\title{
Hyperfine interactions in two-dimensional HgTe topological insulators
}

\author{
Anders Mathias Lunde ${ }^{1,2,3}$ and Gloria Platero ${ }^{1}$ \\ ${ }^{1}$ Instituto de Ciencia de Materiales de Madrid (ICMM), Consejo Superior de Investigaciones Científicas (CSIC), 28049 Madrid, Spain \\ ${ }^{2}$ Instituto de Estructura de la Materia, CSIC, Serrano 123, 28006 Madrid, Spain \\ ${ }^{3}$ Center for Quantum Devices, Niels Bohr Institute, University of Copenhagen, Denmark
}

(Received 17 April 2013; published 5 September 2013)

\begin{abstract}
We study the hyperfine interaction between the nuclear spins and the electrons in a HgTe quantum well, which is the prime experimentally realized example of a two-dimensional topological insulator. The hyperfine interaction is a naturally present, internal source of broken time-reversal symmetry from the point of view of the electrons. The HgTe quantum well is described by the so-called Bernevig-Hughes-Zhang (BHZ) model. The basis states of the BHZ model are combinations of both $S$ - and $P$-like symmetry states, which means that three kinds of hyperfine interactions play a role: (i) the Fermi contact interaction, (ii) the dipole-dipole-like coupling, and (iii) the electron-orbital to nuclear-spin coupling. We provide benchmark results for the forms and magnitudes of these hyperfine interactions within the BHZ model, which give a good starting point for evaluating hyperfine interactions in any HgTe nanostructure. We apply our results to the helical edge states of a HgTe two-dimensional topological insulator and show how their total hyperfine interaction becomes anisotropic and dependent on the orientation of the sample edge within the plane. Moreover, for the helical edge states, the hyperfine interaction due to the $P$-like states can dominate over the $S$-like contribution in certain circumstances.
\end{abstract}

DOI: 10.1103/PhysRevB.88.115411

PACS number(s): 71.70.Jp, 75.75.-c, 73.21.-b, 31.30.Gs

\section{INTRODUCTION}

A topological insulator (TI) hosts gapless surface or edge states, while the bulk of the material has an insulating energy gap. ${ }^{1-4}$ In three-dimensional TIs, the gapless surface states are spin-polarized two-dimensional (2D) Dirac fermions, whereas 2D TIs contain one-dimensional (1D) helical edge states. The helical edge states appear in counterpropagating pairs, and the states with equal energy and opposite wave numbers, $k$ and $-k$, form a Kramers pair. Thus elastic scattering from one helical edge state (HES) to the other one within a pair cannot be induced by time-reversal invariant potentials, e.g., stemming from impurities. ${ }^{5}$ Therefore the transport through a 2D TI is to a large extend ballistic with a quantized conductance of $e^{2} / h$ per pair of HESs. This highlights the central role of time-reversal symmetry in TIs.

Quantized conductance has recently been measured in micrometer-sized samples in $\mathrm{HgTe}$ quantum wells, ${ }^{6-11}$ which to date is the most important experimental demonstration of a 2D TI. Evidence of edge state transport was found in both two-terminal ${ }^{6}$ and multiterminal ${ }^{7}$ devices. Moreover, clever experiments combining the metallic spin Hall effect and a 2D $\mathrm{TI}$ in a HgTe quantum well (QW) demonstrated the connection between the spin and the propagation direction..$^{10}$ However, also deviations from perfect conductance have been observed in longer HgTe devices, ${ }^{6,7,11,12}$ which could stem from, e.g., inelastic scattering mechanisms. ${ }^{13-18}$ The effect of external magnetic fields have also been considered. ${ }^{6,8,19-24}$ The TI state in HgTe QWs was predicted by Bernevig, Hughes, and Zhang $(\mathrm{BHZ})^{25}$ by using a simplified $\mathbf{k} \cdot \mathbf{p}$ model containing states with $S$ - and $P$-like symmetries, respectively. They found that beyond a critical thickness of the $\mathrm{HgTe} \mathrm{QW}$, the TI state would appear as confirmed experimentally. ${ }^{6-9}$ Furthermore, interesting experimental progress on 2D TI properties has also been achieved in InAs/GaSb QW ${ }^{26-28}$ as proposed theoretically. ${ }^{29}$

Hyperfine (HF) interactions between the electron and nuclear spins can play an important role in nanostructures- even though it is often weak. ${ }^{30-33}$ For instance, in quantum dots, HF interactions can limit the coherence of single electronic spins ${ }^{34-37}$ and, moreover, it can even lead to current hysteresis due to bistability of the dynamical nuclear spin polarization. ${ }^{38-41}$ A HF-induced nuclear spin ordering in interacting $1 \mathrm{D}^{42-44}$ and $2 \mathrm{D}^{45,46}$ systems has also been discussed. Most studies consider the so-called contact HF interaction, ${ }^{30-33}$ which is relevant for electrons in orbital states with $S$-like symmetry, e.g., the conduction band in GaAs.

\section{HyPERFINE COUPLING IN THE BHZ BASIS}
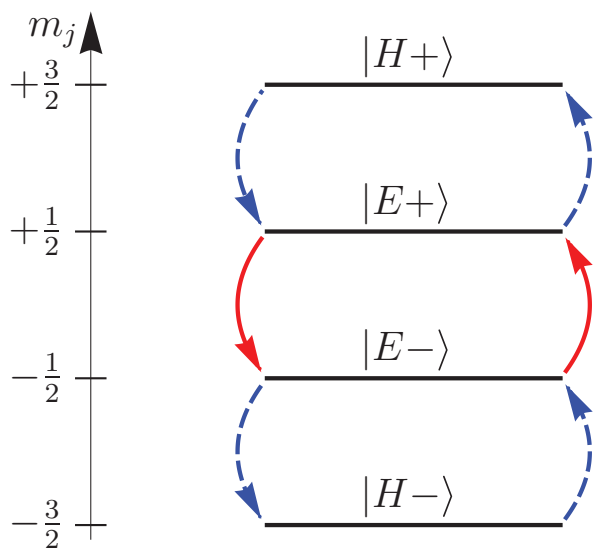

FIG. 1. (Color online) Hyperfine coupling between the BHZ basis states $\{|H+\rangle,|E+\rangle,|E-\rangle,|H-\rangle\}$, which have the total angular momentum projections on the $z$-axis $m_{j}$ as indicated. Every increase (decrease) of $m_{j}$ in the electronic sector is accompanied by a decrease (increase) of a nuclear spin due to angular momentum conservation. Hyperfine interactions due to both $S$ - and $P$-like states connect the two time-reversed blocks of the BHZ model (red full arrows). However, only hyperfine interactions due to $P$-like states connect states within a single time-reversed block (blue dashed arrows). 
However, for $P$-like orbital states - such as the valence band in GaAs - the contact HF interaction is absent. Nevertheless, other anisotropic HF interactions are present for $P$-like states such as the dipole-dipole-like HF interaction, ${ }^{47-51}$ which can play a significant role, e.g., for the decoherence of a hole confined in a quantum dot. ${ }^{47,48,51,52}$

HF interactions and dynamical nuclear spin polarization have also been investigated in the context of integer quantum Hall systems, ${ }^{53-60}$ which contain unidirectional edge states. Here, HF-induced spin-flip transitions between the unidirectional edge states can create nuclear spin polarization locally at the boundary of the $2 \mathrm{D}$ sample. ${ }^{54-60}$ Recently, we have predicted a similar phenomenon for a 2D TI, namely that embedded fixed spins such as the nuclear spins in a 2D TI can polarize locally at the boundary due to a current through the HESs. ${ }^{61}$ Interestingly, the 2D TI with localized spins remains ballistic, ${ }^{61,62}$ except if additional spin-flip mechanisms for the localized spins are present. ${ }^{61}$ However, combining localized spins and Rashba spin-orbit coupling in the 2D TI can produce a conductance change. ${ }^{63-65}$ In the previous works, ${ }^{61-65}$ the interaction between the fixed spins embedded into the $2 \mathrm{D}$ TI and the HESs were modeled phenomenologically. In contrast, here we pay special attention to the detailed forms of the HF interactions within a $2 \mathrm{D} \mathrm{TI}$.

In this paper, we find the different HF interactions within the BHZ model for a HgTe QW. To this end, we take into account both the $S$ - and $P$-like states of the BHZ model, which couple differently to the nuclear spins. We show that all the HF Hamiltonians couple the time-reversed blocks of the $\mathrm{BHZ}$ model. However, only HF interactions relevant for $P$-like states couple states within a time-reversed block as illustrated in Fig. 1. Moreover, we estimate the different HF coupling constants. The derived Hamiltonians are general in the sense that they can be used to find the HF interactions for any kind of nanostructure in a HgTe QW, e.g., quantum dots, ${ }^{66}$ ring structures, ${ }^{67}$ quantum point contacts, ${ }^{68}$ or hole structures. ${ }^{69}$ As an illustrative example, we find the HF interactions for a pair of HESs. Remarkably, the intra-HES transitions coupled to all the nuclear spin components perpendicular to the propagation direction of the HESs. This kind of coupling is unusual compared to, e.g., an ordinary Heisenberg model. Interestingly, the details of the HF interactions depend on the spacial direction of the boundary at which the HESs propagate.

The paper is structured as follows. First, the HF interactions and the BHZ model are outlined in Secs. II and III. Then the HF interactions are found within the BHZ model for the simplest case of a 2D QW (see Sec. IV). From this, we derive the $\mathrm{HF}$ interactions for a given nanostructure in Sec. V. Finally, the HF interactions for the HESs are found and discussed (see Sec. VI). Appendices A-E provide various details for completeness.

\section{THE HYPERFINE INTERACTIONS}

The HF interaction between an electron at position $\mathbf{r}$ with $\operatorname{spin} \mathbf{S}=\left(S_{x}, S_{y}, S_{z}\right)$ and the nuclear spin $\mathbf{I}_{n}=\left(I_{x, n}, I_{y, n}, I_{z, n}\right)$ of the lattice atom at $\mathbf{R}_{n}$ can be derived from the Dirac equation $^{33,50}$ to be (in SI units)

$$
\begin{aligned}
h_{1}^{n} & =\frac{\mu_{0}}{4 \pi} \frac{8 \pi}{3} \gamma_{e} \gamma_{j_{n}} \delta\left(\mathbf{r}_{n}\right) \mathbf{S} \cdot \mathbf{I}_{n}, \\
h_{2}^{n} & =\frac{\mu_{0}}{4 \pi} \gamma_{e} \gamma_{j_{n}} \frac{3\left(\mathbf{e}_{n} \cdot \mathbf{S}\right)\left(\mathbf{e}_{n} \cdot \mathbf{I}_{n}\right)-\mathbf{S} \cdot \mathbf{I}_{n}}{r_{n}^{3}\left(1+r_{c} / r_{n}\right)}, \\
h_{3}^{n} & =\frac{\mu_{0}}{4 \pi} \gamma_{e} \gamma_{j_{n}} \frac{\mathbf{L}_{n} \cdot \mathbf{I}_{n}}{r_{n}^{3}\left(1+r_{c} / r_{n}\right)},
\end{aligned}
$$

where $h_{1}^{n}$ is the Fermi contact interaction, ${ }^{70} h_{2}^{n}$ is the dipoledipole-like coupling between the electrons spin and the nuclear spin, and $h_{3}^{n}$ is the coupling of the electrons orbital momentum $\mathbf{L}_{n}=\mathbf{r}_{n} \times \mathbf{p}$ and the nuclear spin. Here, $\mathbf{r}_{n}=$ $\mathbf{r}-\mathbf{R}_{n}$ is the electrons position relative to the $n$th nucleus, $r_{n} \equiv\left|\mathbf{r}_{n}\right|, \mathbf{e}_{n} \equiv \mathbf{r}_{n} / r_{n}$ and $\mu_{0}$ is the vacuum permeability. The gyromagnetic ratios of the electron $\gamma_{e}$ and the $n$th nuclear spin $\gamma_{j_{n}}$ of the isotope $j$ are, respectively, given by $(e>0)$ $\gamma_{e}=g_{e} \mu_{B} / \hbar$, where $\mu_{B}=e \hbar /\left(2 m_{e}\right)$ is the Bohr magneton and $g_{e} \simeq 2$ the electron $g$ factor, and $\gamma_{j_{n}}=g_{j_{n}} \mu_{N} / \hbar$, where $\mu_{N}=e \hbar /\left(2 m_{p}\right)=\mu_{B} / 1836$ is the nuclear magneton and $g_{j_{n}}$ is the $g$ factor of the $j$ th isotope. Here, $m_{e}$ and $m_{p}$ are the bare electron and proton masses, respectively. ${ }^{71}$ Moreover, $r_{c}$ is a length scale related to the finite size of the nucleus and therefore much smaller than all other length scales in the system. It can be found to be ${ }^{33} r_{c}=Z e^{2} /\left(2 m c^{2}\right) \simeq$ $Z \times 1.5 \mathrm{fm}$, where $Z$ is the number of protons in the nucleus. ${ }^{72}$ Thus the total HF interaction between an electron and all the nuclear spins in the lattice is

$$
\begin{aligned}
H_{\mathrm{HF}} & =H_{\mathrm{HF}, 1}+H_{\mathrm{HF}, 2}+H_{\mathrm{HF}, 3} \\
& =\sum_{n} h_{1}^{n}+\sum_{n} h_{2}^{n}+\sum_{n} h_{3}^{n},
\end{aligned}
$$

where only those lattice points $\mathbf{R}_{n}$ with a nonzero nuclear spin are included in the sum.

Not every atom in a HgTe crystal has a nonzero nuclear spin in contrast to, e.g., GaAs. The amount of stable isotopes with a nonzero spin in $\mathrm{Hg}$ and $\mathrm{Te}$ are about ${ }^{31}$

$$
\begin{array}{r}
17 \% \text { of }{ }^{199} \mathrm{Hg}(\operatorname{spin}-1 / 2), \quad 13 \% \text { of }{ }^{201} \mathrm{Hg}(\operatorname{spin}-3 / 2), \\
1 \% \text { of }{ }^{123} \mathrm{Te}(\operatorname{spin}-1 / 2), \quad 7 \% \text { of }{ }^{125} \mathrm{Te}(\operatorname{spin}-1 / 2) .
\end{array}
$$

Hence, about $19 \%$ of all the atoms in HgTe have a nonzero nuclear spin. By isotope selection processes, this number can be varied somewhat experimentally.

The contact interaction $H_{\mathrm{HF}, 1}$ is the only important $\mathrm{HF}$ interaction for $S$-like states due to their spherical symmetry around the atomic core. On the other hand, $P$-like states vanish at the atomic core and therefore the contact interaction does not affect electrons in those states. In contrast, the two other terms $H_{\mathrm{HF}, 2}$ and $H_{\mathrm{HF}, 3}$ can, indeed, play a role for $P$-like states such as heavy holes. ${ }^{47}$ Moreover, Fischer et ll $^{47}$ found the atomic HF coupling constants to be about one order of magnitude lower for $P$-like compared to $S$-like states in GaAs.

\section{THE BERNEVIG-HUGHES-ZHANG (BHZ) MODEL}

Bernevig, Hughes, and Zhang ${ }^{25}$ constructed a simple model describing the basic physics of a $\mathrm{HgTe} \mathrm{QW}$. The effective $4 \times 4$ BHZ Hamiltonian is derived using $\mathbf{k} \cdot \mathbf{p}$ methods $^{73-75}$ 
and valid for $\mathbf{k}=\left(k_{x}, k_{y}\right)$ close to the $\Gamma$ point, i.e., close to $\mathbf{k}=(0,0)$. The basis states of the model are the two Kramer pairs $|E \pm\rangle$ and $|H \pm\rangle$. Details on the derivation of the $\mathrm{BHZ}$ model are found in Refs. 1,25, and 76. For a 2D QW, the BHZ Hamiltonian is

$$
\mathcal{H}_{0}=\sum_{\mathbf{k}} \mathbf{c}_{\mathbf{k}}^{\dagger} H_{0}(\mathbf{k}) \mathbf{c}_{\mathbf{k}}
$$

where $\mathbf{c}_{\mathbf{k}}^{\dagger}=\left(c_{\mathbf{k}, E+}^{\dagger}, c_{\mathbf{k}, H+}^{\dagger}, c_{\mathbf{k}, E-}^{\dagger}, c_{\mathbf{k}, H_{-}}^{\dagger}\right)$ is a vector of creation operators and

$$
H_{0}(\mathbf{k})=\left(\begin{array}{cc}
h(\mathbf{k}) & \mathbf{0} \\
\mathbf{0} & h^{*}(-\mathbf{k})
\end{array}\right)
$$

with 0 being a zero $2 \times 2$ matrix and

$$
h(\mathbf{k})=\left(\begin{array}{cc}
\varepsilon_{k}+M_{k} & A\left(k_{x}+i k_{y}\right) \\
A\left(k_{x}-i k_{y}\right) & \varepsilon_{k}-M_{k}
\end{array}\right) .
$$

Here, $\varepsilon_{k}=-D k^{2}, M_{k}=M_{0}-B k^{2}$, and $k \equiv \sqrt{k_{x}^{2}+k_{y}^{2}}$ have been introduced. ${ }^{77}$ The parameters $A, B, D$, and $M_{0}$ depend on the QW geometry. ${ }^{1,25}$ Importantly, varying the QW width changes the sign of $M_{0}$, which in turn makes the system go from a nontopological to a topological state with HESs. ${ }^{25}$

The Hamiltonian (4a) a priori has periodic boundary conditions and thereby does not contain any edges. By introducing boundaries into the model, it is possible to derive explicitly the HESs in the TI state of the QW. ${ }^{78,79}$ This will be discussed further in Sec. VIA.

Within the envelope function approximation ${ }^{73-75}$ the states of the BHZ model are

$$
\begin{aligned}
|E+\rangle & =f_{E \Gamma_{6}}(z)\left|\Gamma_{6},+1 / 2\right\rangle+f_{E \Gamma_{8}}(z)\left|\Gamma_{8},+1 / 2\right\rangle, \\
|H+\rangle & =f_{H}(z)\left|\Gamma_{8},+3 / 2\right\rangle, \\
|E-\rangle & =f_{E \Gamma_{6}}(z)\left|\Gamma_{6},-1 / 2\right\rangle+f_{E \Gamma_{8}}(z)\left|\Gamma_{8},-1 / 2\right\rangle, \\
|H-\rangle & =f_{H}(z)\left|\Gamma_{8},-3 / 2\right\rangle,
\end{aligned}
$$

where $f_{i}(z)$ are the transverse envelope functions in the $z$ direction perpendicular to the $2 \mathrm{D} \mathrm{QW}$ and $\left|\Gamma_{i}, m_{j}\right\rangle$ are the lattice periodic functions ${ }^{80}$ at $\mathbf{k}=0$ for the $\Gamma_{i}$ band with projection $m_{j}$ of the total angular momentum, $\mathbf{J}=\mathbf{L}+\mathbf{S}$, on the $z$ axis. Here, $\mathbf{S}$ is the electron spin and $\mathbf{L}$ is the orbital angular momentum (see Appendix A). The time-reversal operator $\Theta$ connects states within a Kramer pair $(\Theta|E \pm\rangle=$ $\mp|E \mp\rangle$ and $\Theta|H \pm\rangle=\mp|H \mp\rangle$ ), and the two blocks in $H_{0}(\mathbf{k})$ (4b) are related by time reversal. Here, we choose phase conventions of the envelope functions such that time-reversed partners have equal envelope functions. Moreover, $f_{E \Gamma_{6}}$ and $f_{H}$ are chosen real, whereas $f_{E \Gamma_{8}}$ is chosen purely imaginary. (Appendix A gives more details on the envelope functions and the lattice periodic functions.)

The states $|E \pm\rangle$ are seen to be mixtures of the $S$-like $\Gamma_{6}$ band and the $P$-like $\Gamma_{8}$ band with $m_{j}= \pm 1 / 2$, whereas $|H \pm\rangle$ consist only of the $P$-like $\Gamma_{8}$ band with $m_{j}= \pm 3 / 2$. Hence the states have a definite total angular momentum projection,

$$
J_{z}|E \pm\rangle= \pm \frac{1}{2} \hbar|E \pm\rangle \quad \text { and } \quad J_{z}|H \pm\rangle= \pm \frac{3}{2} \hbar|H \pm\rangle,
$$

but $|E \pm\rangle$ are not eigenstates of $\mathbf{J}^{2}$.

The HF interactions can only induce transitions between states with a difference of angular momentum projection of one unit: $m_{j}-m_{j^{\prime}}= \pm 1$. Therefore we can already at this point see that only particular combinations of the BHZ states can be connected by HF interactions as seen in Fig. 1. Furthermore, it is evident that $\mathrm{HF}$ interactions relevant for both $S$ - and $P$-like states need to be included to have a full description of the HF interactions in a HgTe TI.

The real-space basis functions of $\mathcal{H}_{0}(4 \mathrm{a})$ for the $2 \mathrm{D} \mathrm{QW}$ with periodic boundary conditions are

$$
\begin{aligned}
\varphi_{\mathbf{k}, E \pm}(\mathbf{r})= & \frac{\sqrt{v_{a}}}{\sqrt{L_{x} L_{y}}} e^{i\left(k_{x} x+k_{y} y\right)} \\
& \times\left[f_{E \Gamma_{6}}(z) u_{\Gamma_{6}, \pm \frac{1}{2}}(\mathbf{r})+f_{E \Gamma_{8}}(z) u_{\Gamma_{8}, \pm \frac{1}{2}}(\mathbf{r})\right] \\
\varphi_{\mathbf{k}, H \pm}(\mathbf{r})= & \frac{\sqrt{v_{a}}}{\sqrt{L_{x} L_{y}}} e^{i\left(k_{x} x+k_{y} y\right)} f_{H}(z) u_{\Gamma_{8}, \pm \frac{3}{2}}(\mathbf{r})
\end{aligned}
$$

where $\mathbf{r}=(x, y, z), L_{x}\left(L_{y}\right)$ is the QW length in the $x(y)$ direction, and $u_{\Gamma_{i}, m_{j}}(\mathbf{r}) \equiv\left\langle\mathbf{r} \mid \Gamma_{i}, m_{j}\right\rangle$ are the real-space lattice periodic functions at $\mathbf{k}=0$. Moreover, we have included the atomic volume ${ }^{81} v_{a}$ explicitly here as it is often done for HF related calculations. ${ }^{47-50}$ It depends on the choice of the individual normalization of the envelope functions and the lattice periodic functions, respectively, if $v_{a}$ should be included explicitly, ${ }^{30}$ as discussed in Appendix B.

\section{HYPERFINE INTERACTIONS WITHIN THE BHZ MODEL}

Next, we find the HF interactions within the BHZ model by using the states (7) for a $2 \mathrm{D} \mathrm{QW}$ with periodic boundary conditions. As we shall see, these results are useful, since they allow us to find the HF interactions for any nanostructure created in a HgTe QW (see Sec. V).

\section{A. Outline of the way to find the hyperfine interaction matrix elements}

The HF interactions (1) are local in space on the atomic scale, so the important part of the wave function with respect to the HF interactions is the behavior around the nucleus. Hence, in the envelope function approximation, it is the rapidly varying lattice periodic functions $u_{\Gamma_{i}, m_{j}}(\mathbf{r})$ that play the central role, whereas the slowly varying envelope functions only are multiplicative factors at the atomic nucleus, as we shall see below.

We set out to find the HF interactions

$$
\mathcal{H}_{\mathrm{HF}, i}=\sum_{\mathbf{k}, \mathbf{k}^{\prime}} \sum_{\substack{v, v^{\prime}=E, H \\ \tau \tau^{\prime}= \pm}}\left\langle\varphi_{\mathbf{k} v \tau}\left|H_{\mathrm{HF}, i}\right| \varphi_{\mathbf{k}^{\prime} v^{\prime} \tau^{\prime}}\right\rangle c_{\mathbf{k} v \tau}^{\dagger} c_{\mathbf{k}^{\prime} v^{\prime} \tau^{\prime}}
$$

for $i=1,2,3$ in the basis (7), i.e., for $\varphi_{\mathbf{k}, v \pm}(\mathbf{r})$ with $v=E, H$. We begin by describing the general way that we find the HF interaction matrix elements $\left\langle\varphi_{\mathbf{k} v \tau}\left|H_{\mathrm{HF}, i}\right| \varphi_{\mathbf{k}^{\prime} v^{\prime} \tau^{\prime}}\right\rangle$. To this end, the integration over the entire system volume $\mathcal{V}$ is rewritten as a sum of integrals over each unit cell $m$ of volume $v_{u c}^{(m)}$, i.e.,

$$
\int_{\mathcal{V}} d \mathbf{r}(\cdots)=\sum_{\mathcal{R}_{m}} \int_{v_{u c}^{(m)}} d \rho(\cdots) .
$$

This should be understood in the following way: every space point $\mathbf{r}$ can be reached by first a Bravais lattice vector 
$\mathcal{R}_{m} \equiv\left(\mathcal{X}_{m}, \mathcal{Y}_{m}, \mathcal{Z}_{m}\right)$ and then a vector $\rho$ within the $m$ th unit cell, i.e., $\mathbf{r}=\boldsymbol{\mathcal { R }}_{m}+\boldsymbol{\rho}$. The superscript $(m)$ on the unit cell volume $v_{u c}^{(m)}$ indicates that the integral is over the $m$ th unit cell. Thus the matrix element is

$$
\begin{aligned}
& \left\langle\varphi_{\mathbf{k} v \tau}\left|H_{\mathrm{HF}, i}\right| \varphi_{\mathbf{k}^{\prime} v^{\prime} \tau^{\prime}}\right\rangle \\
& \quad=\sum_{n} \sum_{\mathcal{R}_{m}} \int_{v_{u c}^{(m)}} d \rho \varphi_{\mathbf{k} v \tau}^{*}\left(\mathcal{R}_{m}+\boldsymbol{\rho}\right) h_{i}^{n} \varphi_{\mathbf{k}^{\prime} v^{\prime} \tau^{\prime}}\left(\boldsymbol{\mathcal { R }}_{m}+\boldsymbol{\rho}\right) .
\end{aligned}
$$

Here, one sum is over all unit cells $\boldsymbol{\mathcal { R }}_{m}$, whereas the other sum is only over those atoms at position $\mathbf{R}_{n}$ with a nonzero nuclear spin. ${ }^{82}$ To proceed, we take $v=v^{\prime}=H$ as an illustrative example and obtain

$$
\begin{aligned}
\left\langle\varphi_{\mathbf{k} H \tau}\left|H_{\mathrm{HF}, i}\right| \varphi_{\mathbf{k}^{\prime} H \tau^{\prime}}\right\rangle \simeq & \frac{v_{a}}{L_{x} L_{y}} \sum_{n} \sum_{\mathcal{R}_{m}} e^{i\left(\mathbf{k}^{\prime}-\mathbf{k}\right) \cdot \mathcal{R}_{m \perp}}\left|f_{H}\left(\mathcal{Z}_{m}\right)\right|^{2} \\
& \times \int_{v_{u c}^{(m)}} d \rho u_{\Gamma_{8}, \tau 3 / 2}^{*}(\rho) h_{i}^{n} u_{\Gamma_{8}, \tau^{\prime} 3 / 2}(\rho),
\end{aligned}
$$

where we have used the slow variation of the envelope functions on the atomic scale, $f_{H}\left(\rho_{z}+\mathcal{Z}_{m}\right) \simeq f_{H}\left(\mathcal{Z}_{m}\right)$, and the lattice periodicity of the lattice periodic functions, e.g., $u_{\Gamma_{8}, \tau \frac{3}{2}}\left(\mathcal{R}_{m}+\boldsymbol{\rho}\right)=u_{\Gamma_{8}, \tau \frac{3}{2}}(\boldsymbol{\rho})$ for all $\boldsymbol{\mathcal { R }}_{m}$. Here, $\mathcal{R}_{m \perp} \equiv\left(\mathcal{X}_{m}, \mathcal{Y}_{m}\right)$, and the integral over $\rho$ is over the $m$ th unit cell, whereas $h_{i}^{n}$ is for the $n$th nuclei. For a specific nuclear spin $n$, we now include only the integral over that particular unit cell containing the $n$th nuclear spin, since the HF interactions are local in space. In other words, if the nuclei spin $n$ is not inside the integration volume of the unit cell $m$, then the contribution is neglected, ${ }^{83}$ i.e.,

$$
\begin{aligned}
\left\langle\varphi_{\mathbf{k} H \tau}\left|H_{\mathrm{HF}, i}\right| \varphi_{\mathbf{k}^{\prime} H \tau^{\prime}}\right\rangle= & \frac{v_{a}}{L_{x} L_{y}} \sum_{n} e^{i\left(\mathbf{k}^{\prime}-\mathbf{k}\right) \cdot \mathcal{R}_{n \perp}}\left|f_{H}\left(\mathcal{Z}_{n}\right)\right|^{2} \\
& \times \int_{v_{u c}} d \rho u_{\Gamma_{8}, \tau 3 / 2}^{*}(\rho) h_{i}^{n} u_{\Gamma_{8}, \tau^{\prime} 3 / 2}(\rho),
\end{aligned}
$$

where the unit cell integral now is independent of the unit cell position $\boldsymbol{\mathcal { R }}_{n}$. The sum is only over the lattice nuclei at $\mathbf{R}_{n}$ with a nonzero nuclear spin. Therefore the system does not have discrete translational symmetry, so the sum cannot simply be made into an integral. Hence, the matrix elements are not diagonal in $\mathbf{k}$ due to the nuclear spins at random lattice points.

In order to proceed, we need to evaluate the integral of the lattice periodic function over the unit cell in Eq. (12). To this end, the symmetry of the lattice periodic functions are important; the contact interaction $H_{\mathrm{HF}, 1}$ is zero for $P$-like states, since they vanish on the atomic center, while matrix elements of $H_{\mathrm{HF}, i}$ for $i=2,3$ vanish for $S$-like states due to their spherical symmetry. Here, we approximate the lattice periodic functions by a linear combination of atomic orbitals (LCAO) as ${ }^{47,84}$

$$
\begin{aligned}
& u_{\Gamma_{6}, m_{j}}(\mathbf{r})=N_{\Gamma_{6}, m_{j}}\left[\alpha_{\mathrm{Te}} \Psi_{\Gamma_{6}, m_{j}}^{\mathrm{Te}}\left(\mathbf{r}+\frac{\mathbf{d}}{2}\right)-\alpha_{\mathrm{Hg}} \Psi_{\Gamma_{6}, m_{j}}^{\mathrm{Hg}}\left(\mathbf{r}-\frac{\mathbf{d}}{2}\right)\right], \\
& u_{\Gamma_{8}, m_{j}}(\mathbf{r})=N_{\Gamma_{8}, m_{j}}\left[\alpha_{\mathrm{Te}} \Psi_{\Gamma_{8}, m_{j}}^{\mathrm{Te}}\left(\mathbf{r}+\frac{\mathbf{d}}{2}\right)+\alpha_{\mathrm{Hg}} \Psi_{\Gamma_{8}, m_{j}}^{\mathrm{Hg}}\left(\mathbf{r}-\frac{\mathbf{d}}{2}\right)\right],
\end{aligned}
$$

where $\Psi_{\Gamma_{i}, m_{j}}^{\mathrm{Te}}$ and $\Psi_{\Gamma_{i}, m_{j}}^{\mathrm{Hg}}$ are atomic-like wave functions centered on the $\mathrm{Te}$ and $\mathrm{Hg}$ atoms, respectively, and $\mathbf{r}$ is only within a single two-atomic primitive unit cell of $\mathrm{HgTe}$ centered at $\mathbf{r}=0$. The atomic wave functions inherit the symmetry of the band ${ }^{47,84}$ as indicated by the index $\Gamma_{i}, m_{j}$. The atoms are connected by the vector $\mathbf{d}$, and the constants $N_{\Gamma_{i}, m_{j}}$ are determined by the lattice periodic function normalization $\int_{v_{u c}} d \mathbf{r}\left|u_{\Gamma_{i}, m_{j}}(\mathbf{r})\right|^{2}=2$, see, e.g., Eq. (B4). The electron sharing within the unit cell is described by $\alpha_{\mathrm{Te}(\mathrm{Hg})}$, which fulfill $\left|\alpha_{\mathrm{Te}}\right|^{2}+\left|\alpha_{\mathrm{Hg}}\right|^{2}=1 .^{85}$

The LCAO approach (13) now facilitates evaluation of the unit cell integral in the matrix elements $\left\langle\varphi_{\mathbf{k} v \tau}\left|H_{\mathrm{HF}, i}\right| \varphi_{\mathbf{k}^{\prime} v^{\prime} \tau^{\prime}}\right\rangle$. Consider, e.g., the unit cell integral in Eq. (12) for a nonzero spin on the $n$th $\mathrm{Hg}$ nucleus located on $\rho=\mathbf{d} / 2$, i.e.,

$$
\begin{array}{rl}
\int_{v_{u c}} & d \rho u_{\Gamma_{8}, \tau 3 / 2}^{*}(\boldsymbol{\rho}) h_{i}^{n} u_{\Gamma_{8}, \tau^{\prime} 3 / 2}(\boldsymbol{\rho}) \\
\simeq & N_{\Gamma_{8}, \tau 3 / 2}^{*} N_{\Gamma_{8}, \tau^{\prime} 3 / 2}\left|\alpha_{\mathrm{Hg}}\right|^{2} \\
\quad & \times \int_{v_{u c}} d \rho\left[\Psi_{\Gamma_{8}, \tau 3 / 2}^{\mathrm{Hg}}(\boldsymbol{\rho}-\mathbf{d} / 2)\right]^{*} h_{i}^{n} \Psi_{\Gamma_{8}, \tau^{\prime} 3 / 2}^{\mathrm{Hg}}(\boldsymbol{\rho}-\mathbf{d} / 2),
\end{array}
$$

where only the important contribution of the atomic wave functions centered on the $\mathrm{Hg}$ atom is included. In other words, integrals involving atomic wave functions centered on different atoms are neglected. Fischer et al. ${ }^{47}$ estimated that these nonlocal contributions are two to three orders of magnitude smaller for GaAs - even for the long-ranged potentials in $h_{2,3}^{n}$ in Eqs. (1b) and (1c).

Thus we have now outlined how to find the matrix elements $\left\langle\varphi_{\mathbf{k} H \tau}\left|H_{\mathrm{HF}, i}\right| \varphi_{\mathbf{k}^{\prime} H \tau^{\prime}}\right\rangle$ for all three kinds of $\mathrm{HF}$ interactions (1). The matrix elements of the types $\left\langle\varphi_{\mathbf{k} E \tau}\left|H_{\mathrm{HF}, i}\right| \varphi_{\mathbf{k}^{\prime} E \tau^{\prime}}\right\rangle$ and $\left\langle\varphi_{\mathbf{k} E \tau}\left|H_{\mathrm{HF}, i}\right| \varphi_{\mathbf{k}^{\prime} H \tau^{\prime}}\right\rangle$ follow the same lines as above. The essential ingredients are the locality of the HF interactions, the periodicity of $u_{\Gamma_{i}, m_{j}}(\mathbf{r})$ and the slowly varying envelope functions. Next, we find the three HF interactions (1) within the BHZ model.

\section{B. The contact $\mathrm{HF}$ interaction for $S$-like states}

Now we find the contact $\mathrm{HF}$ interaction $H_{\mathrm{HF}, 1}$ Eq. (1a) within the BHZ basis (7). We begin by noting that $\left\langle\varphi_{\mathbf{k} H \tau}\left|H_{\mathrm{HF}, 1}\right| \varphi_{\mathbf{k}^{\prime} H \tau^{\prime}}\right\rangle=0$ and $\left\langle\varphi_{\mathbf{k} E \tau}\left|H_{\mathrm{HF}, 1}\right| \varphi_{\mathbf{k}^{\prime} H \tau^{\prime}}\right\rangle=0$, since the contact interaction is only nonzero at the atomic center $\left(h_{1}^{n} \propto \delta\left(\mathbf{r}-\mathbf{R}_{n}\right)\right)$, where the $\Gamma_{8} P$-like atomic orbitals vanish. Hence, only the $\Gamma_{6} S$-like part of the $\varphi_{\mathbf{k}^{\prime} E \tau^{\prime}}(\mathbf{r})$ states leads to nonzero matrix elements of $H_{\mathrm{HF}, 1}$. Using the approach in Sec. IV A to find the matrix elements, we get

$$
\begin{aligned}
\left\langle\varphi_{\mathbf{k} E \tau}\left|H_{\mathrm{HF}, 1}\right| \varphi_{\mathbf{k}^{\prime} E \tau^{\prime}}\right\rangle= & \frac{v_{a}}{L_{x} L_{y}} \sum_{n} e^{i\left(\mathbf{k}^{\prime}-\mathbf{k}\right) \cdot \mathcal{R}_{n \perp}}\left|f_{E \Gamma_{6}}\left(\mathcal{Z}_{n}\right)\right|^{2} \\
& \times \int_{v_{u c}} d \rho u_{\Gamma_{6}, \tau 1 / 2}^{*}(\boldsymbol{\rho}) h_{1}^{n} u_{\Gamma_{6}, \tau^{\prime} 1 / 2}(\boldsymbol{\rho})
\end{aligned}
$$

for $\tau, \tau^{\prime}= \pm$. The $\Gamma_{6}$ states $u_{\Gamma_{6}, \pm 1 / 2}(\mathbf{r})$ simply factorize into a spin and an orbital part as $u_{\Gamma_{6},+(-) 1 / 2}(\mathbf{r})=u_{\Gamma_{6}}(\mathbf{r})|\uparrow(\downarrow)\rangle$, see, e.g., Eq. (A2). Using this and the explicit form of the contact 
interaction $h_{1}^{n}$ in Eq. (1a), we readily obtain

$$
\begin{aligned}
\left\langle\varphi_{\mathbf{k} E \tau}\left|H_{\mathrm{HF}, 1}\right| \varphi_{\mathbf{k}^{\prime} E \tau^{\prime}}\right\rangle= & \frac{1}{L_{x} L_{y}} \sum_{n} e^{i\left(\mathbf{k}^{\prime}-\mathbf{k}\right) \cdot \mathcal{R}_{n \perp}} A_{S, j_{n}}\left(\mathcal{Z}_{n}\right) \\
& \times \frac{1}{\hbar}\left[\tau \frac{1}{2} I_{z, n} \delta_{\tau, \tau^{\prime}}+\frac{1}{2} I_{\tau^{\prime}, n} \delta_{\tau,-\tau^{\prime}}\right],
\end{aligned}
$$

where $I_{ \pm, n} \equiv I_{x, n} \pm i I_{y, n}$ are the raising and lowering nuclear spin operators. In analog to the case of a quantum dot, ${ }^{30,47}$ we here introduce the position dependent contact HF coupling as $^{86}$

$$
A_{S, j_{n}}\left(\mathcal{Z}_{n}\right) \equiv v_{a}\left|f_{E \Gamma_{6}}\left(\mathcal{Z}_{n}\right)\right|^{2} A_{S, j_{n}}^{\text {Atomic }},
$$

which includes the atomic contact HF coupling

$$
A_{S, j_{n}}^{\text {Atomic }} \equiv \frac{2 \mu_{0}}{3} g_{e} \mu_{B} g_{j_{n}} \mu_{N}\left|u_{\Gamma_{6}}\left(\mathbf{R}_{n}\right)\right|^{2}
$$

for the nuclear spin at site $n$ of isotope $j$. Here, $A_{S, j_{n}}\left(\mathcal{Z}_{n}\right)$ depends on the real-space position of the nuclear spin. In contrast, $A_{S, j_{n}}^{\text {Atomic }}$ does not depend on the nuclear position, since it can be given in terms of the atomic orbital $\Psi_{\Gamma_{6}}^{j_{n}}$ by using Eq. (13) as $A_{S, j_{n}}^{\text {Atomic }} \propto\left|u_{\Gamma_{6}}\left(\mathbf{R}_{n}\right)\right|^{2} \simeq\left|N_{\Gamma_{6}, 1 / 2}\right|^{2}\left|\alpha_{j_{n}}\right|^{2}\left|\Psi_{\Gamma_{6}}^{j_{n}}(0)\right|^{2}$, i.e., $A_{S, j_{n}}^{\text {Atomic }}$ only depends on the nuclear isotope type $j_{n}$ at site $n$. Moreover, at the present level of approximation, we can freely replace the Bravais lattice vector $\boldsymbol{\mathcal { R }}_{n}$ by the actual position of a nuclear spin within the $n$th unit cell in the envelope functions in Eq. (16) due to their slow variation. Finally, we arrive at the HF contact interaction in the BHZ basis as

$$
\mathcal{H}_{\mathrm{HF}, 1}=\sum_{n} \sum_{\mathbf{k}, \mathbf{k}^{\prime}} \frac{e^{i\left(\mathbf{k}^{\prime}-\mathbf{k}\right) \cdot \mathcal{R}_{n \perp}}}{L_{x} L_{y}} \mathbf{c}_{\mathbf{k}}^{\dagger} \tilde{H}_{\mathrm{HF}, 1} \mathbf{c}_{\mathbf{k}^{\prime}}
$$

where $\mathbf{c}_{\mathbf{k}}^{\dagger}=\left(c_{\mathbf{k}, E+}^{\dagger}, c_{\mathbf{k}, H+}^{\dagger}, c_{\mathbf{k}, E-}^{\dagger}, c_{\mathbf{k}, H-}^{\dagger}\right)$ and $^{87}$

$$
\tilde{H}_{\mathrm{HF}, 1}=\frac{1}{2 \hbar} A_{S, j_{n}}\left(\mathcal{Z}_{n}\right)\left(\begin{array}{cccc}
I_{z, n} & 0 & I_{-, n} & 0 \\
0 & 0 & 0 & 0 \\
I_{+, n} & 0 & -I_{z, n} & 0 \\
0 & 0 & 0 & 0
\end{array}\right) \text {. }
$$

The sum is only over nonzero nuclear spins. Therefore it is now clear that the contact HF interaction contains elements $\propto I_{ \pm, n}$, which connect the time-reversed blocks in the BHZ Hamiltonian (4b). Moreover, as illustrated in Fig. 1, only the $|E \pm\rangle$ states are connected by $H_{\mathrm{HF}, 1}$, since only these states contain a $S$-like symmetry part. In in Table I, estimates of the atomic con-

TABLE I. Estimates of the atomic contact HF couplings $A_{S, j_{n}}^{\text {Atomic }}$, Eq. (18), and the atomic $P$-like HF couplings $A_{P, j_{n}}^{\text {Atom }}$, Eq. (26), in $\mathrm{HgTe}$ for the naturally present isotopes with nonzero spin, see Eq. (3). The HF couplings for $S$-like states are seen to be about one order of magnitude larger than for $P$-like states. The sign of the HF couplings stems from the sign of the nuclear $g$ factors. See Appendix D for details of these estimates.

\begin{tabular}{lcccc}
\hline \hline & ${ }^{199} \mathrm{Hg}$ & ${ }^{201} \mathrm{Hg}$ & ${ }^{123} \mathrm{Te}$ & ${ }^{125} \mathrm{Te}$ \\
\hline$A_{S, j_{n}}^{\text {Atomic }}(\mu \mathrm{eV})$ & 4.1 & -1.5 & -49 & -59 \\
$A_{P, j_{n}}^{\text {Atoc }}(\mu \mathrm{eV})$ & 0.6 & -0.2 & -6.0 & -7.2 \\
\hline \hline
\end{tabular}

tact HF couplings $A_{S, j_{n}}^{\text {Atomic }}$ are given for the stable isotopes of $\mathrm{HgTe}$ with nonzero nuclear spin (see Appendix D for details).

\section{The HF interactions for $\boldsymbol{P}$-like states}

Next, we find the HF interactions within the BHZ basis (7) for $H_{\mathrm{HF}, 2}$ and $H_{\mathrm{HF}, 3}$, Eqs. (1b) and (1c), which are relevant for the $P$-like states. To begin with, we argue that the $\Gamma_{6} S$-like states-part of the $E \pm$ states-do not contribute to the matrix elements $\left\langle\varphi_{\mathbf{k} E \tau}\left|H_{\mathrm{HF}, i}\right| \varphi_{\mathbf{k}^{\prime} E \tau^{\prime}}\right\rangle$ and $\left\langle\varphi_{\mathbf{k} E \tau}\left|H_{\mathrm{HF}, i}\right| \varphi_{\mathbf{k}^{\prime} H \tau^{\prime}}\right\rangle$ for $i=$ 2,3. (In contrast, the $\Gamma_{8} P$-like part of $E \pm$ does contribute to these elements as will be shown below.) To understand this, the HF matrix elements are written in terms of the unit cell integrals over the atomic-like wave functions as outlined in Sec. IV A. Firstly, for the dipole-dipole-like HF interaction (1b), we have

$$
\int_{v_{u c}} d \rho\left[\Psi_{\Gamma_{6}, m_{j}}^{\mathrm{Hg} / \mathrm{Te}}(\rho \mp \mathbf{d} / 2)\right]^{*} h_{2}^{n} \Psi_{\Gamma_{6}, m_{j}^{\prime}}^{\mathrm{Hg} / \mathrm{Te}}(\rho \mp \mathbf{d} / 2)=0
$$

due to the rotational symmetry of the $S$-like orbitals around the atomic core. ${ }^{72}$ Secondly, we have

$$
\int_{v_{u c}} d \rho\left[\Psi_{\Gamma_{6}, m_{j}}^{\mathrm{Hg} / \mathrm{Te}}(\rho \mp \mathbf{d} / 2)\right]^{*} h_{2}^{n} \Psi_{\Gamma_{8}, m_{j}^{\prime}}^{\mathrm{Hg} / \mathrm{Te}}(\rho \mp \mathbf{d} / 2)=0
$$

due to opposite parities of the $S$ - and $P$-like orbitals. ${ }^{88}$ The same matrix elements containing $h_{3}^{n}$ instead of $h_{2}^{n}$ are also zero, because the $S$-like states have zero orbital momentum, i.e., $\mathbf{L}_{n} \Psi_{\Gamma_{6}, m_{j}}^{\mathrm{Hg} / \mathrm{Te}}(\boldsymbol{\rho})=0$.

Therefore only $P$-like states contribute, so we are now left with (see Sec. IV A)

$$
\begin{aligned}
\left\langle\varphi_{\mathbf{k} E \tau}\left|H_{\mathrm{HF}, i}\right| \varphi_{\mathbf{k}^{\prime} E \tau^{\prime}}\right\rangle= & \frac{v_{a}}{L_{x} L_{y}} \sum_{n} e^{i\left(\mathbf{k}^{\prime}-\mathbf{k}\right) \cdot \mathcal{R}_{n \perp}}\left|f_{E \Gamma_{8}}\left(\mathcal{Z}_{n}\right)\right|^{2} \\
& \times \int_{v_{u c}} d \rho u_{\Gamma_{8}, \tau 1 / 2}^{*}(\boldsymbol{\rho}) h_{i}^{n} u_{\Gamma_{8}, \tau^{\prime} 1 / 2}(\boldsymbol{\rho}), \\
\left\langle\varphi_{\mathbf{k} H \tau}\left|H_{\mathrm{HF}, i}\right| \varphi_{\mathbf{k}^{\prime} H \tau^{\prime}}\right\rangle= & \frac{v_{a}}{L_{x} L_{y}} \sum_{n} e^{i\left(\mathbf{k}^{\prime}-\mathbf{k}\right) \cdot \mathcal{R}_{n \perp}}\left|f_{H}\left(\mathcal{Z}_{n}\right)\right|^{2} \\
& \times \int_{v_{u c}} d \rho u_{\Gamma_{8}, \tau 3 / 2}^{*}(\boldsymbol{\rho}) h_{i}^{n} u_{\Gamma_{8}, \tau^{\prime} 3 / 2}(\boldsymbol{\rho}), \\
\left\langle\varphi_{\mathbf{k} E \tau}\left|H_{\mathrm{HF}, i}\right| \varphi_{\mathbf{k}^{\prime} H \tau^{\prime}}\right\rangle= & \frac{v_{a}}{L_{x} L_{y}} \sum_{n} e^{i\left(\mathbf{k}^{\prime}-\mathbf{k}\right) \cdot \mathcal{R}_{n \perp}} f_{E \Gamma_{8}}^{*}\left(\mathcal{Z}_{n}\right) f_{H}\left(\mathcal{Z}_{n}\right) \\
& \times \int_{v_{u c}} d \rho u_{\Gamma_{8}, \tau 1 / 2}^{*}(\rho) h_{i}^{n} u_{\Gamma_{8}, \tau^{\prime} 3 / 2}(\boldsymbol{\rho}),
\end{aligned}
$$

and $\left\langle\varphi_{\mathbf{k} H \tau}\left|H_{\mathrm{HF}, i}\right| \varphi_{\mathbf{k}^{\prime} E \tau^{\prime}}\right\rangle=\left\langle\varphi_{\mathbf{k}^{\prime} E \tau^{\prime}}\left|H_{\mathrm{HF}, i}\right| \varphi_{\mathbf{k} H \tau}\right\rangle^{*}$, where $i=$ 2,3 and $\tau, \tau^{\prime}= \pm$. Using the LCAO approach (13), the unit cell integrals over the lattice periodic functions now become integrals over the atomic-like wave functions as in Eq. (14). We write the atomic wave functions as a product of a radial part $R^{\mathrm{Hg} / \mathrm{Te}}(r)$ and an angular part $\mathbb{Y}_{\Gamma_{8}, m_{j}}(\theta, \phi)$, i.e., $\Psi_{\Gamma_{8}, m_{j}}^{\mathrm{Hg} / \mathrm{Te}}(\mathbf{r})=$ $R^{\mathrm{Hg} / \mathrm{Te}}(r) \mathbb{Y}_{\Gamma_{8}, m_{j}}(\theta, \phi)$, using spherical coordinates $(r, \theta, \phi)$ with the nucleus in the center. Since the integrals are over the two-atomic unit cell volume, they do not a priori factorize into a product of radial and angular integrals. However, due to 
the $1 / r^{3}$ dependence of $h_{i}^{n}(i=2,3)$, the important part of the unit cell integrals are numerically within one or two Bohr radii $a_{0}$ from the atomic core, which is certainly within the unit cell volume. Therefore it is a good approximation to write the unit cell integrals [e.g., Eq. (14)] as

$$
\begin{array}{rl}
\int_{v_{u c}} & d \rho\left[\Psi_{\Gamma_{8}, m_{j}}^{\mathrm{Hg}}(\rho-\mathbf{d} / 2)\right]^{*} h_{i}^{n} \Psi_{\Gamma_{8}, m_{j}^{\prime}}^{\mathrm{Hg}}(\boldsymbol{\rho}-\mathbf{d} / 2) \\
\simeq & \int_{0}^{r_{\max }} d r r^{2} \int_{0}^{2 \pi} d \phi \int_{0}^{\pi} d \theta \sin (\theta)\left[\Psi_{\Gamma_{8}, m_{j}}^{\mathrm{Hg}}(\mathbf{r})\right]^{*} h_{2}^{n} \Psi_{\Gamma_{8}, m_{j}^{\prime}}^{\mathrm{Hg}}(\mathbf{r}),
\end{array}
$$

where the specific choice of $r_{\max } \gtrsim a_{0}$ is not important for the numerical value of the integral. ${ }^{89}$ Therefore we are now left with an essentially atomic physics problem, where the integral separates into a product of a radial and an angular part. The radial part is

$$
\left\langle\frac{1}{r^{3}}\right\rangle_{r}^{\kappa} \equiv \int_{0}^{r_{\max }} d r r^{2}\left|R^{\kappa}(r)\right|^{2} \frac{1}{r^{3}\left(1+\frac{r_{c}}{r}\right)},
$$

which is the same for all the matrix elements of $h_{2}^{n}$ and $h_{3}^{n}$ and only depends on the type of atom $\kappa=\mathrm{Hg}$, Te. Due to the smallness of the nuclear length scale $r_{c}$, it is not significant for the magnitude of $\left\langle 1 / r^{3}\right\rangle_{r}^{\kappa}$. ${ }^{72}$ Using the radial integral (25), we introduce the atomic $P$-like HF coupling for isotope $j$ (at site $n)$ as

$$
A_{P, j_{n}}^{\text {Atomic }} \equiv \frac{\mu_{0}}{4 \pi} g_{e} \mu_{B} g_{j_{n}} \mu_{N}\left(N_{\Gamma_{8}}\right)^{2}\left|\alpha_{j_{n}}\right|^{2}\left\langle\frac{1}{r^{3}}\right\rangle_{r}^{j_{n}},
$$

which are estimated to be about one order of magnitude smaller than the atomic contact HF couplings $A_{S, j_{n}}^{\text {Atomic }}$ (18), see Table I. Here, it makes sense to have a common atomic HF coupling for the dipole-dipole-like coupling $h_{2}^{n}$ and the orbital to nuclear-spin coupling $h_{3}^{n}$, since the normalization constants for the LCAO lattice functions (13) are numerically approximately equal, $N_{\Gamma_{8}, 3 / 2} \simeq N_{\Gamma_{8}, 1 / 2} \equiv N_{\Gamma_{8}}$, as discussed in Appendix D. ${ }^{90}$ Moreover, we also use that $N_{\Gamma_{i}, m_{j}}$ are independent of the sign of $m_{j}$, see Eq. (D6). Calculating the angular integrals as discussed in Appendix $\mathrm{C}$, the matrix elements (23) for the dipole-dipole-like $\mathrm{HF}$ interaction $H_{\mathrm{HF}, 2}$ become

$$
\begin{aligned}
\left\langle\varphi_{\mathbf{k} E \tau}\left|H_{\mathrm{HF}, 2}\right| \varphi_{\mathbf{k}^{\prime} E \tau^{\prime}}\right\rangle= & \sum_{n} \frac{1}{L_{x} L_{y}} e^{i\left(\mathbf{k}^{\prime}-\mathbf{k}\right) \cdot \mathcal{R}_{n \perp}} A_{P, j_{n}}^{E E} \frac{1}{\hbar} \\
& \times\left[-\tau \delta_{\tau^{\prime}, \tau} \frac{1}{15} I_{z, n}-\delta_{\tau^{\prime},-\tau} \frac{2}{15} I_{-\tau, n}\right], \\
\left\langle\varphi_{\mathbf{k} H \tau}\left|H_{\mathrm{HF}, 2}\right| \varphi_{\mathbf{k}^{\prime} H \tau^{\prime}}\right\rangle= & \sum_{n} \frac{e^{i\left(\mathbf{k}^{\prime}-\mathbf{k}\right) \cdot \mathcal{R}_{n \perp}}}{L_{x} L_{y}} \frac{-\tau A_{P, j_{n}}^{H H} \delta_{\tau, \tau^{\prime}} I_{z, n}}{5 \hbar},
\end{aligned}
$$

$$
\left\langle\varphi_{\mathbf{k} E \tau}\left|H_{\mathrm{HF}, 2}\right| \varphi_{\mathbf{k}^{\prime} H \tau^{\prime}}\right\rangle=\sum_{n} \frac{e^{i\left(\mathbf{k}^{\prime}-\mathbf{k}\right) \cdot \mathcal{R}_{n \perp}}}{L_{x} L_{y}} \frac{-A_{P, j_{n}}^{E H} \delta_{\tau^{\prime}, \tau} I_{\tau, n}}{5 \sqrt{3} \hbar}
$$

where we introduce the position dependent $P$-like HF couplings as

$$
\begin{aligned}
& A_{P, j_{n}}^{H H} \equiv v_{a}\left|f_{H}\left(\mathcal{Z}_{n}\right)\right|^{2} A_{P, j_{n}}^{\text {Atomic }}, \\
& A_{P, j_{n}}^{E E} \equiv v_{a}\left|f_{E \Gamma_{8}}\left(\mathcal{Z}_{n}\right)\right|^{2} A_{P, j_{n}}^{\text {Atomic }}, \\
& A_{P, j_{n}}^{E H} \equiv v_{a} f_{E \Gamma_{8}}^{*}\left(\mathcal{Z}_{n}\right) f_{H}\left(\mathcal{Z}_{n}\right) A_{P, j_{n}}^{\text {Atomic }},
\end{aligned}
$$

and $A_{P, j_{n}}^{H E}=\left[A_{P, j_{n}}^{E H}\right]^{*}$. In comparison, for the contact $\mathrm{HF}$ interaction only a single position dependent HF coupling was introduced in Eq. (17). Here, the explicit dependence on the position $\mathcal{Z}_{n}$ of the nuclear spin has been suppressed in the notation for simplicity, i.e., $A_{P, j_{n}}^{v v^{\prime}}\left(\mathcal{Z}_{n}\right)=A_{P, j_{n}}^{v v^{\prime}}$. Similarly, the matrix elements for the $\mathrm{HF}$ interaction $H_{\mathrm{HF}, 3}$ between the electronic orbital momentum and the nuclear spins become

$$
\begin{aligned}
\left\langle\varphi_{\mathbf{k} E \tau}\left|H_{\mathrm{HF}, 3}\right| \varphi_{\mathbf{k}^{\prime} E \tau^{\prime}}\right\rangle= & \sum_{n} \frac{1}{L_{x} L_{y}} e^{i\left(\mathbf{k}^{\prime}-\mathbf{k}\right) \cdot \mathcal{R}_{n \perp}} A_{P, j_{n}}^{E E} \\
& \times \frac{1}{\hbar}\left[\delta_{\tau, \tau^{\prime}} \frac{1}{3} \tau I_{z, n}+\delta_{-\tau, \tau^{\prime}} \frac{2}{3} I_{-\tau, n}\right], \\
\left\langle\varphi_{\mathbf{k} H \tau}\left|H_{\mathrm{HF}, 3}\right| \varphi_{\mathbf{k}^{\prime} H \tau^{\prime}}\right\rangle= & \sum_{n} \frac{e^{i\left(\mathbf{k}^{\prime}-\mathbf{k}\right) \cdot \mathcal{R}_{n \perp}}}{L_{x} L_{y}} \frac{A_{P, j_{n}}^{H H} \delta_{\tau, \tau^{\prime}} \tau I_{z, n}}{\hbar} \\
\left\langle\varphi_{\mathbf{k} E \tau}\left|H_{\mathrm{HF}, 3}\right| \varphi_{\mathbf{k}^{\prime} H \tau^{\prime}}\right\rangle= & \sum_{n} \frac{e^{i\left(\mathbf{k}^{\prime}-\mathbf{k}\right) \cdot \mathcal{R}_{n \perp}}}{L_{x} L_{y}} \frac{A_{P, j_{n}}^{E H} \delta_{\tau, \tau^{\prime}} I_{\tau, n}}{\sqrt{3} \hbar} .
\end{aligned}
$$

It is noteworthy that the heavy-hole-like states $H \pm$ only couple diagonally $\left(\tau=\tau^{\prime}\right)$ or Ising-like in Eqs. (27b) and (29b) in agreement with Ref. 47. Physically, this is because the $H \pm$ states have a difference of total angular momentum projection larger than one, $\left|m_{j}-m_{j^{\prime}}\right|>1$. Moreover, the coupling between the states $E \pm$ in Eqs. (27a) and (29a) is essentially like the coupling between the light hole states $\left|\Gamma_{8}, \pm 1 / 2\right\rangle$, since the $S$-like states do not contribute to the matrix elements of $H_{\mathrm{HF}, 2}$ and $H_{\mathrm{HF}, 3}$. For these matrix elements between the $E \pm$ states, the off-diagonal elements $\left(\tau=-\tau^{\prime}\right)$ are a factor of 2 larger than the diagonal elements $\left(\tau=\tau^{\prime}\right)$ in accordance with Ref. 51 .

Using the matrix elements in Eqs. (27) and (29), we now finally arrive at the $\mathrm{HF}$ interactions relevant for the $P$-like states in the basis (7) as

$$
\mathcal{H}_{\mathrm{HF}, i}=\sum_{n} \sum_{\mathbf{k}, \mathbf{k}^{\prime}} \frac{e^{i\left(\mathbf{k}^{\prime}-\mathbf{k}\right) \cdot \mathcal{R}_{n \perp}}}{L_{x} L_{y}} \mathbf{c}_{\mathbf{k}}^{\dagger} \tilde{H}_{\mathrm{HF}, i} \mathbf{c}_{\mathbf{k}^{\prime}}
$$

for $i=2,3$, where

$$
\tilde{H}_{\mathrm{HF}, 2}=\frac{1}{5 \hbar}\left(\begin{array}{cccc}
-\frac{1}{3} A_{P, j_{n}}^{E E} I_{z, n} & -\frac{1}{\sqrt{3}} A_{P, j_{n}}^{E H} I_{+, n} & -\frac{2}{3} A_{P, j_{n}}^{E E} I_{-, n} & 0 \\
-\frac{1}{\sqrt{3}} A_{P, j_{n}}^{H E} I_{-, n} & -A_{P, j_{n}}^{H H} I_{z, n} & 0 & 0 \\
-\frac{2}{3} A_{P, j_{n}}^{E E} I_{+, n} & 0 & \frac{1}{3} A_{P, j_{n}}^{E E} I_{z, n} & -\frac{1}{\sqrt{3}} A_{P, j_{n}}^{E H} I_{-, n} \\
0 & 0 & -\frac{1}{\sqrt{3}} A_{P, j_{n}}^{H E} I_{+, n} & A_{P, j_{n}}^{H H} I_{z, n}
\end{array}\right) \text {, }
$$


and

$$
\tilde{H}_{\mathrm{HF}, 3}=-5 \tilde{H}_{\mathrm{HF}, 2}
$$

such that the total HF interaction for the $P$-like states becomes

$$
\tilde{H}_{\mathrm{HF}, P}=\tilde{H}_{\mathrm{HF}, 2}+\tilde{H}_{\mathrm{HF}, 3}=-4 \tilde{H}_{\mathrm{HF}, 2} .
$$

Just as the contact HF interaction (20), the $P$-like HF interaction connects the time-reversed blocks by connecting the $E \pm$ states. Moreover, the $P$-like HF interaction connects the states within the time-reversed blocks (e.g., $E+$ and $\mathrm{H+}$ ) in contrast to the contact $\mathrm{HF}$ interaction, see Fig. 1.

Interestingly, the sign of the dipole-dipole-like HF interaction (31a) is opposite to the contact HF interaction (20) and to the orbital to nuclear-spin coupling (31b). However, since the elements of $\tilde{H}_{\mathrm{HF}, 3}$ are larger than those of $\tilde{H}_{\mathrm{HF}, 2}$ in absolute value, the total $\mathrm{HF}$ interaction for the $P$-like states (32) ends up having the same sign as the contact HF interaction.

\section{HYPERFINE INTERACTIONS FOR A NANOSTRUCTURE IN A HgTe QUANTUM WELL}

Now, we show how the HF interactions for any nanostructure in a $\mathrm{HgTe}$ QW can be derived from our results in Eqs. (20) and (31) for a $\mathrm{HgTe} \mathrm{QW}$ with periodic boundary conditions. Examples of such structures are quantum dots, ${ }^{66}$ mesoscopic rings, ${ }^{67}$ point contacts, ${ }^{68}$ and antidots. ${ }^{69}$

For a given nanostructure, the envelope wave functions are needed in order to find its HF interactions within the BHZ framework. Utilizing the Peierls substitution $\left(k_{x}, k_{y}\right)=$ $-i\left(\partial_{x}, \partial_{y}\right)$ in the BHZ Hamiltonian (4b), the envelope functions $\Phi_{\eta}\left(\mathbf{r}_{\perp}\right)$ can be found by solving

$$
\left[H_{0}\left(-i \partial_{x},-i \partial_{y}\right)+V\left(\mathbf{r}_{\perp}\right)\right] \Phi_{\eta}\left(\mathbf{r}_{\perp}\right)=E_{\eta} \Phi_{\eta}\left(\mathbf{r}_{\perp}\right),
$$

where $V\left(\mathbf{r}_{\perp}\right)$ is the potential confining the nanostructure, ${ }^{91}$ $\mathbf{r}_{\perp} \equiv(x, y)$ and $\eta$ is a collection of quantum numbers to be specified for a concrete situation. ${ }^{66,67,69,78,92}$ Terms related to bulk inversion asymmetry, ${ }^{8,22}$ Rashba spin-orbit coupling ${ }^{13,76}$ and/or magnetic fields ${ }^{19,21}$ can also be included here. The envelope function is given by 93

$$
\Phi_{\eta}\left(\mathbf{r}_{\perp}\right)=\left(\begin{array}{c}
\phi_{\eta, E+}\left(\mathbf{r}_{\perp}\right) \\
\phi_{\eta, H+}\left(\mathbf{r}_{\perp}\right) \\
\phi_{\eta, E-}\left(\mathbf{r}_{\perp}\right) \\
\phi_{\eta, H-}\left(\mathbf{r}_{\perp}\right)
\end{array}\right)
$$

such that the entire wave function including the lattice periodic functions is

$$
\psi_{\eta}(\mathbf{r})=\sqrt{v_{a}} \sum_{\zeta=E \pm, H \pm} \phi_{\eta, \zeta}\left(\mathbf{r}_{\perp}\right)\langle\mathbf{r} \mid \zeta\rangle,
$$

where $\langle\mathbf{r} \mid E \pm\rangle=f_{E \Gamma_{6}}(z) u_{\Gamma_{6}, \pm \frac{1}{2}}(\mathbf{r})+f_{E \Gamma_{8}}(z) u_{\Gamma_{8}, \pm \frac{1}{2}}(\mathbf{r})$ and $\langle\mathbf{r} \mid H \pm\rangle=f_{H}(z) u_{\Gamma_{8}, \pm \frac{3}{2}}(\mathbf{r})$. For instance, the $2 \mathrm{D} \mathrm{QW}$ with periodic boundary conditions simply has the envelope functions $e^{i\left(k_{x} x+k_{y} y\right)}(1,0,0,0)^{T} / \sqrt{L_{x} L_{y}}$, etc., see Eq. (7).

To find the HF interactions (1) for a given nanostructure with envelope wave function $\Phi_{\eta}\left(\mathbf{r}_{\perp}\right)$ (33), the same idea of separation of length scales as in Sec. IV is used: the HF interactions act on the atomic length scale such that the slowly varying envelope functions only become multiplicative factors in the HF interactions for the nanostructure. Thus we find

$$
\mathcal{H}_{\mathrm{HF}, i}=\sum_{n} \sum_{\eta, \eta^{\prime}}\left[\Phi_{\eta}\left(\boldsymbol{\mathcal { R }}_{n \perp}\right)\right]^{\dagger} \tilde{H}_{\mathrm{HF}, i} \Phi_{\eta^{\prime}}\left(\boldsymbol{\mathcal { R }}_{n \perp}\right) c_{\eta}^{\dagger} c_{\eta^{\prime}},
$$

where $\tilde{H}_{\mathrm{HF}, i}$ are the $4 \times 4$ matrices found in Eqs. (20), (31), and (32) for the contact $(i=1)$ and $P$-like $(i=2,3, P) \mathrm{HF}$ interactions, respectively. The sum is only over the atomic sites $n$ with a nonzero nuclear spin. The Bravais lattice vector $\mathcal{R}_{n \perp} \equiv\left(\mathcal{X}_{n}, \mathcal{Y}_{n}\right)$ pointing to the unit cell containing the $n$th nuclear spin can freely be interchanged by the atomic position $\mathbf{R}_{n}$ of the nuclear spin due to the slow variation of the envelope functions on the atomic scale. ${ }^{82}$

In situations with time-reversal symmetry, the states appear in Kramers pairs of equal energy. The BHZ model in Eq. (4) is constructed such that Kramers pairs appear as equal energy solutions of the upper and lower $2 \times 2$ blocks in $H_{0}$. Thus $\phi_{\eta, E-}=\phi_{\eta, H-}=0$ for one of the two states in a Kramers pair and vice versa, which simplifies the algebraic burden of finding $\Phi_{\eta} \cdot{ }^{66,67,69,78}$ The two states in a Kramers pair are sometimes referred to as spin up and down, since the upper (lower) block only consists of orbital states with positive (negative) total angular momentum projection, see Eq. (6). If other time-reversal invariant interactions such as the Rashba spin-orbit coupling ${ }^{13,76}$ or bulk inversion asymmetry terms ${ }^{8}$ are included into the $\mathrm{BHZ}$ model for a nanostructure, then the states still appear in Kramers pairs-even though the Hamiltonian is not necessarily in block diagonal form anymore. In this case, the general formula (35) for the HF interactions remains valid, since only the slowly varying envelope functions are affected. Here the index for the Kramers pair is included in $\eta$. Thus we have now provided the general form of the $\mathrm{HF}$ interactions in the BHZ model for a given nanostructure in Eq. (35). Below we illustrate its use by an example.

\section{HYPERFINE INTERACTIONS FOR A PAIR OF HELICAL EDGE STATES}

Next, we deal with the HF interactions for a pair of HESs in a HgTe 2D TI QW.

\section{A. The helical edge states along the $y$ axis}

To find the HF interactions, we first give the envelope wave functions for a pair of HESs. These can be found by introducing a boundary in the $\mathrm{BHZ}$ model and requiring that the envelope functions vanish at the boundary. ${ }^{78}$ For a semi-infinite halfplane restricted to $x>0$ and periodic boundary condition in the $y$ direction, $k_{y}$ is still a good quantum number. The HESs envelope functions running along the $y$ direction become ${ }^{78}$ (see Fig. 2)

$$
\Psi_{y, k_{y}}^{\varsigma}(x, y)=\frac{1}{\sqrt{L_{y}}} e^{i k_{y} y} \mathfrak{h}_{k_{y}}^{\varsigma}(x) \chi_{y}^{\varsigma} \text { for } \varsigma=u, d
$$

with the energy dispersions $E_{k_{y}}^{u}=E_{0}+\hbar v_{0} k_{y}$ and $E_{k_{y}}^{d}=E_{0}-\hbar v_{0} k_{y}$, respectively. The dispersions are exactly 


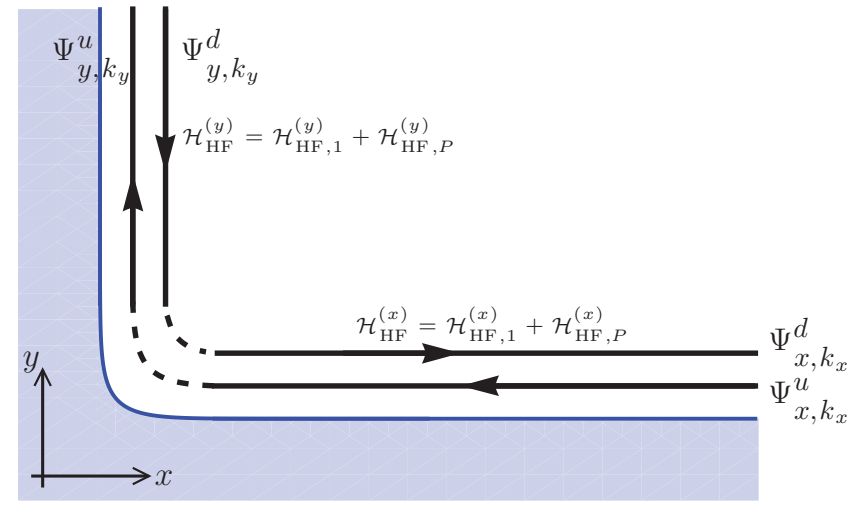

FIG. 2. (Color online) The helical edge states along the $y$ axis, Eq. (36), and along the $x$ axis, Eq. (42), are connected by $k_{y} \leftrightarrow-k_{x}$. Moreover, they differ by an imaginary unit $i$ in the $E$ spinor components [compare Eqs. (37) and (43)]. This leads to interesting differences in their HF Hamiltonians (see the main text). For illustrative purposes, the HESs are drawn side by side even though equal energy HESs in fact are on top of each other.

linear in the semi-infinite half plane model used here. ${ }^{79}$ Both the velocity $v_{0}=-\sqrt{B^{2}-D^{2}}|A| /(\hbar B)$ and $E_{0}=-M_{0} D / B$ are positive for realistic parameters ${ }^{94}$ and $L_{y}$ is the length of the edge. The spinor parts of the HESs are independent of $k_{y}$ and given by

$$
\chi_{y}^{u}=\mathfrak{n}\left(\begin{array}{c}
-i \frac{A}{|A|} \\
\frac{\sqrt{B^{2}-D^{2}}}{B-D} \\
0 \\
0
\end{array}\right), \quad \chi_{y}^{d}=\mathfrak{n}\left(\begin{array}{c}
0 \\
0 \\
+i \frac{A}{|A|} \\
\frac{\sqrt{B^{2}-D^{2}}}{B-D}
\end{array}\right)
$$

with the normalization factor $\mathfrak{n}=\sqrt{(B-D) / 2 B}$. The transverse part of the HESs are $\mathfrak{h}_{k_{y}}^{u}(x)=g_{+k_{y}}(x)$ and $\mathfrak{h}_{k_{y}}^{d}(x)=$ $g_{-k_{y}}(x)$, where

$$
g_{k_{y}}(x)=\sqrt{\frac{2 \lambda_{1} \lambda_{2}\left(\lambda_{1}+\lambda_{2}\right)}{\left(\lambda_{1}-\lambda_{2}\right)^{2}}}\left(e^{-\lambda_{1} x}-e^{-\lambda_{2} x}\right),
$$

with the $k_{y}$-dependence inside $\lambda_{1}$ and $\lambda_{2}$ as

$$
\begin{aligned}
& \lambda_{1}=\frac{1}{\sqrt{B^{2}-D^{2}}}\left(\frac{|A|}{2}+\sqrt{W_{k_{y}}}\right), \\
& \lambda_{2}=\frac{1}{\sqrt{B^{2}-D^{2}}}\left(\frac{|A|}{2}-\sqrt{W_{k_{y}}}\right) .
\end{aligned}
$$

Here, $\lambda_{2}^{-1}$ determines the decay length scale of the HES into the bulk and

$$
\begin{aligned}
W_{k_{y}}= & {\left[\frac{A^{2}}{4}-\frac{M_{0}}{B}\left(B^{2}-D^{2}\right)\right]+\frac{D|A| \sqrt{B^{2}-D^{2}}}{B} k_{y} } \\
& +\left(B^{2}-D^{2}\right) k_{y}^{2} .
\end{aligned}
$$

The HESs only exist in the topological regime of the BHZ model where $M_{0} / B>0$. The explicit forms above were derived under the assumption $0 \leqslant M_{0} / B \leqslant A^{2} /\left(4 B^{2}\right)$, where $\lambda_{1,2}$ are purely real. ${ }^{78,95}$ This is the relevant regime for the realistic parameters ${ }^{1,94}$ for $2 \mathrm{D} \mathrm{TI}$ in a $\mathrm{HgTe} \mathrm{QW}$ of width 61 or $70 \AA$.

Using the time-reversal properties of the basis states of the BHZ model (as discussed in Appendix A), it is seen explicitly that $\Psi_{y, k_{y}}^{u}(x, y)$ and $\Psi_{y,-k_{y}}^{d}(x, y)$ constitute a Kramers pair, since they are connected by the time-reversal operator $\Theta$ as $\Theta \Psi_{y, k_{y}}^{u}(x, y)=-\Psi_{y,-k_{y}}^{d}(x, y)$ and $\Theta \Psi_{y, k_{y}}^{d}(x, y)=$ $\Psi_{y,-k_{y}}^{u}(x, y)$. Often ${ }^{1,8} \Psi_{y, k_{y}}^{u}\left(\Psi_{y, k_{y}}^{d}\right)$ is referred to as the spin-up (spin-down) edge state, since it only consists of states with positive (negative) total angular momentum projection, see Eq. (37).

\section{B. Hyperfine interactions for the helical edge states along the $y$ axis}

The HF interactions are now readily found by inserting the envelope HESs along the $y$ axis (36) into the general HF interaction formula (35) for any structure in a $\mathrm{HgTe} \mathrm{QW}$. Using Eq. (20), the contact HF interaction becomes

$$
\begin{aligned}
\mathcal{H}_{\mathrm{HF}, 1}^{(y)}= & \frac{1}{2 \hbar} \frac{B-D}{2 B} \sum_{k_{y} k_{y}^{\prime}} \sum_{n} \frac{e^{i\left(k_{y}^{\prime}-k_{y}\right) \mathcal{Y}_{n}}}{L_{y}} A_{S, j_{n}}\left(\mathcal{Z}_{n}\right) \\
& \times\left[\Lambda_{k_{y}, k_{y}^{\prime}}^{\left(\mathcal{X}_{n}\right)} I_{z, n} c_{k_{y} u}^{\dagger} c_{k_{y}^{\prime} u}-\Lambda_{-k_{y},-k_{y}^{\prime}}^{\left(\mathcal{X}_{n}\right)} I_{z, n} c_{k_{y} d}^{\dagger} c_{k_{y}^{\prime} d}\right. \\
& \left.-\Lambda_{k_{y},-k_{y}^{\prime}}^{\left(\mathcal{X}_{n}\right)} I_{-, n} c_{k_{y} u}^{\dagger} c_{k_{y}^{\prime} d}-\Lambda_{-k_{y}, k_{y}^{\prime}}^{\left(\mathcal{X}_{n}\right)} I_{+, n} c_{k_{y} d}^{\dagger} c_{k_{y}^{\prime} u}\right]
\end{aligned}
$$

where $c_{k_{y} \varsigma}^{\dagger}\left(c_{k_{y} \varsigma}\right)$ are the creation (annihilation) operators for the HESs $\Psi_{y, k_{y}}^{\varsigma}(x, y)(36)$. We have emphasized in the notation that $\mathcal{H}_{\mathrm{HF}, 1}^{(y)}$ is for HESs along the $y$ axis. The product of the transverse parts of the HESs at the nuclear $\operatorname{spin} n$ is introduced as

$$
\Lambda_{k_{y}, k_{y}^{\prime}}^{\left(\mathcal{X}_{n}\right)} \equiv g_{k_{y}}^{*}\left(\mathcal{X}_{n}\right) g_{k_{y}^{\prime}}\left(\mathcal{X}_{n}\right)
$$

and includes the only dependence of $\mathcal{X}_{n}$ in $\mathcal{H}_{\mathrm{HF}, 1}^{(y)}$. Here we see that the contact HF interactions can produce transitions between the HESs $\Psi_{y, k_{y}}^{u}(x, y)$ and $\Psi_{y, k_{y}^{\prime}}^{d}(x, y)$ at the expense of a change in a nuclear spin state. In particular, elastic transitions within the Kramers pair $\Psi_{y, k_{y}}^{u}(x, y)$ and $\Psi_{y,-k_{y}}^{d}(x, y)$ are possible. This is just as if the HESs were spin- $1 / 2$ as used, e.g., in Refs. 61,62, and 64. Hence, from the point of view of the electrons in the HESs the time reversal symmetry is broken. Of course, the composed system of electrons and nuclear spins is time-reversal invariant, since any system can be made time-reversal invariant by expanding it sufficiently. ${ }^{96}$

The HF interaction due to the $P$-like states, $\mathcal{H}_{\mathrm{HF}, P}^{(y)}=\mathcal{H}_{\mathrm{HF}, 2}^{(y)}+\mathcal{H}_{\mathrm{HF}, 3}^{(y)}$, is similarly found by inserting 
$\Psi_{y, k_{y}}^{\varsigma}$ (36) into Eq. (35) and using $\tilde{H}_{\mathrm{HF}, P}(32)$, i.e.,

$$
\begin{aligned}
\mathcal{H}_{\mathrm{HF}, P}^{(y)}= & \frac{2}{15 \hbar} \sum_{n} \sum_{k_{y}, k_{y}^{\prime}} \frac{e^{i\left(k_{y}^{\prime}-k_{y}\right) \mathcal{Y}_{n}}}{L_{y}}\left\{-2 \frac{B-D}{B} A_{P, j_{n}}^{E E}\left[\Lambda_{k_{y},-k_{y}^{\prime}}^{\left(\mathcal{X}_{n}\right)} I_{-, n} c_{k_{y} u}^{\dagger} c_{k_{y}^{\prime} d}+\Lambda_{-k_{y}, k_{y}^{\prime}}^{\left(\mathcal{X}_{n}\right)} I_{+, n} c_{k_{y} d}^{\dagger} c_{k_{y}^{\prime} u}\right]\right. \\
& \left.+\left[-\frac{2 \sqrt{3} A \sqrt{B^{2}-D^{2}}}{|A| B} \operatorname{Im}\left(A_{P, j_{n}}^{E H}\right) I_{x, n}+\frac{(B-D) A_{P, j_{n}}^{E E}+3(B+D) A_{P, j_{n}}^{H H}}{B} I_{z, n}\right]\left[\Lambda_{k_{y}, k_{y}^{\prime}}^{\left(\mathcal{X}_{X^{\prime}}\right)} c_{k_{y} u}^{\dagger} c_{k_{y}^{\prime} u}-\Lambda_{-k_{y},-k_{y}^{\prime}}^{\left(\mathcal{X}_{n}\right)} c_{k_{y} d}^{\dagger} c_{k_{y}^{\prime} d}\right]\right\},
\end{aligned}
$$

where the dependence on $\mathcal{Z}_{n}$ is inside the HF couplings $A_{P, j_{n}}^{X Y}$ (28). Here, we used the rewritings $i\left[I_{+, n} A_{P, j_{n}}^{E H}-I_{-, n} A_{P, j_{n}}^{H E}\right]=-2 I_{x, n} \operatorname{Im}\left(A_{P, j_{n}}^{E H}\right) \quad$ and $i\left[I_{+, n} A_{P, j_{n}}^{H E}-I_{-, n} A_{P, j_{n}}^{E H}\right]=+2 I_{x, n} \operatorname{Im}\left(A_{P, j_{n}}^{E H}\right)$, which build on the fact that $A_{P, j_{n}}^{E H}=i \operatorname{Im}\left(A_{P, j_{n}}^{E H}\right)$ due to the phase conventions of $f_{E \Gamma_{8}}$ as purely imaginary and $f_{H}$ as real. This HF interaction also permits transitions between the two HESs-especially within the Kramers pair-just as the contact HF interaction (39). The terms $\propto c_{k \varsigma}^{\dagger} c_{k^{\prime} \varsigma}$ in the HF interaction (41) affect transitions within a single HES. These are more unusual than their counterparts in the contact HF interaction (39), since they do not only contain terms involving $I_{z, n}$, but also $I_{x, n}$. Hence the HF interaction (41) due to the $P$-like states has terms like a $I_{x} S_{z}$ coupling, which are not present in, e.g., a Heisenberg model. These terms $\propto I_{x, n} c_{k \zeta}^{\dagger} c_{k^{\prime} \varsigma}$ stem from the fact that the $\mathrm{HF}$ interactions due to the $P$-like states (31) couple the states $|H \pm\rangle$ and $|E \pm\rangle$ within a single time-reversed block of $H_{0}$. In order to shine more light on the form of the HF interactions (39) and (41), they are given in Appendix E in terms of nondiagonal edge state spin operators using the picture of spin- $1 / 2$ HESs.

\section{Hyperfine interactions for helical edge states along the $x$ axis: curious differences}

The HF interactions presented above are for HESs running along the $y$ axis. Now, we find various interesting differences in the HF interactions for HESs running along the $x$ axis.

The HESs are found in the same way as in Sec. VIA. The only difference is that we consider the HESs localized near a boundary given by the $x$ axis instead of the $y$ axis, i.e., we study the semi-infinite half-plane defined by $y>0$. The HESs along the $x$ axis are given by

$$
\Psi_{x, k_{x}}^{\varsigma}(x, y)=\frac{1}{\sqrt{L_{x}}} e^{i k_{x} x} \mathfrak{g}_{k_{x}}^{\varsigma}(y) \chi_{x}^{\varsigma} \quad \text { for } \quad \varsigma=u, d
$$

Here, $\mathfrak{g}_{k_{x}}^{u}(y)=g_{-k_{x}}(y)$ and $\mathfrak{g}_{k_{x}}^{d}(y)=g_{+k_{x}}(y)$ in terms of $g_{k}$ in Eq. (38) and the spinor parts are

$$
\chi_{x}^{u}=\mathfrak{n}\left(\begin{array}{c}
\frac{A}{|A|} \\
\frac{\sqrt{B^{2}-D^{2}}}{B-D} \\
0 \\
0
\end{array}\right), \quad \chi_{x}^{d}=\mathfrak{n}\left(\begin{array}{c}
0 \\
0 \\
\frac{A}{|A|} \\
\frac{\sqrt{B^{2}-D^{2}}}{B-D}
\end{array}\right),
$$

i.e., the imaginary unit $i$ does not appear in the $E \pm$ components of the spinors as for the HESs along the $y$ axis, see Eq. (37). This is the mathematical origin of the differences between the HF interactions for the HESs in the two directions. These HESs also appear in Kramers pairs $\left(\Psi_{x, k_{x}}^{u}\right.$ and $\left.\Psi_{x,-k_{x}}^{d}\right)$ and $\Psi_{x, k_{x}}^{u}\left(\Psi_{x, k_{x}}^{d}\right)$ is referred to as spin-up (spin-down). The spin-up HES $\Psi_{x, k_{x}}^{u}$ has negative velocity such that $E_{k_{x}}^{u}=E_{0}-\hbar v_{0} k_{x}$, while the spin-down HES has positive velocity, i.e., $E_{k_{x}}^{d}=E_{0}+\hbar v_{0} k_{x}$. Hence the velocities of the HESs along the $x$ and $y$ axes have opposite signs, such that spin- $\varsigma$ always travels the same way along the boundary, see Fig. 2. Therefore it is natural that $k_{x}$ has to be exchanged by $-k_{y}$ to connect the HESs in the two perpendicular directions.

By inserting the HESs along the $x$-axis Eq. (42) into Eq. (35), the contact HF interaction becomes

$$
\begin{aligned}
\mathcal{H}_{\mathrm{HF}, 1}^{(x)}= & \frac{1}{2 \hbar} \frac{B-D}{2 B} \sum_{k_{x} k_{x}^{\prime}} \sum_{n} \frac{e^{i\left(k_{x}^{\prime}-k_{x}\right) \mathcal{X}_{n}}}{L_{x}} A_{S, j_{n}}\left(\mathcal{Z}_{n}\right) \\
& \times\left[\Lambda_{-k_{x},-k_{x}^{\prime}}^{\left(\mathcal{Y}_{n}\right)} I_{z, n} c_{k_{x} u}^{\dagger} c_{k_{x}^{\prime} u}-\Lambda_{k_{x}, k_{x}^{\prime}}^{\left(\mathcal{Y}_{n}\right)} I_{z, n} c_{k_{x} d}^{\dagger} c_{k_{x}^{\prime} d}\right. \\
& \left.+\Lambda_{-k_{x}, k_{x}^{\prime}}^{\left(\mathcal{Y}_{n}\right)} I_{-, n} c_{k_{x} u}^{\dagger} c_{k_{x}^{\prime} d}+\Lambda_{k_{x},-k_{x}^{\prime}}^{\left(\mathcal{Y}_{n}\right)} I_{+, n} c_{k_{x} d}^{\dagger} c_{k_{x}^{\prime} u}\right]
\end{aligned}
$$

where $\Lambda_{k_{x}, k_{x}^{\prime}}^{\left(\mathcal{Y}_{n}\right)}=g_{k_{x}}^{*}\left(\mathcal{Y}_{n}\right) g_{k_{x}^{\prime}}\left(\mathcal{Y}_{n}\right)$. Interestingly, the sign of the terms producing inter HES transitions is opposite to the one in $\mathcal{H}_{\mathrm{HF}, 1}^{(y)}$ (39). This difference stems from the imaginary unit $i$ in $\chi_{y}^{5}$ (37), which is absent in $\chi_{x}^{5}$ (43). Moreover, the sign of $k_{x}$ and $k_{y}$ is opposite in the $\Lambda$ functions for $\mathcal{H}_{\mathrm{HF}, 1}^{(x)}$ (44) and $\mathcal{H}_{\mathrm{HF}, 1}^{(y)}$ (39), respectively. This is natural in order to maintain the propagation direction of the HES-spin $\varsigma=u, d$, see Fig. 2.

The HF interaction due to the $P$-like states for the HESs at the $x$ axis becomes

$$
\begin{aligned}
\mathcal{H}_{\mathrm{HF}, P}^{(x)}= & \frac{2}{15 \hbar} \sum_{n} \sum_{k_{x}, k_{x}^{\prime}} \frac{e^{i\left(k_{x}^{\prime}-k_{x}\right) \mathcal{X}_{n}}}{L_{x}}\left\{+2 \frac{B-D}{B} A_{P, j_{n}}^{E E}\left[\Lambda_{-k_{x}, k_{x}^{\prime}}^{\left(\mathcal{Y}_{n}\right)} I_{-, n} c_{k_{x} u}^{\dagger} c_{k_{x}^{\prime} d}+\Lambda_{k_{x},-k_{x}^{\prime}}^{\left(\mathcal{Y}_{n}\right)} I_{+, n} c_{k_{x} d}^{\dagger} c_{k_{x}^{\prime} u}\right]\right. \\
& +\left[-\frac{2 \sqrt{3} A \sqrt{B^{2}-D^{2}}}{|A| B} \operatorname{Im}\left(A_{P, j_{n}}^{E H}\right) I_{y, n}+\frac{\left.\left.(B-D) A_{P, j_{n}}^{E E}+3(B+D) A_{P, j_{n}}^{H H} I_{z, n}\right]\left[\Lambda_{-k_{x},-k_{x}^{\prime}}^{\left(\mathcal{Y}_{n}\right)} c_{k_{x} u}^{\dagger} c_{k_{x}^{\prime} u}-\Lambda_{k_{x}, k_{x}^{\prime}}^{\left(\mathcal{Y}_{n}\right)} c_{k_{x} d}^{\dagger} c_{k_{x}^{\prime} d}\right]\right\},}{B},\right.
\end{aligned}
$$


where the inter HES transition terms again have an opposite overall sign compared to $\mathcal{H}_{\mathrm{HF}, P}^{(y)}$ (41). Another noteworthy difference is the exchange of the terms $I_{x, n} c_{k_{y} \varsigma}^{\dagger} c_{k_{y}^{\prime} s}$ in $\mathcal{H}_{\mathrm{HF}, P}^{(y)}$ by $I_{y, n} c_{k_{x} \varsigma}^{\dagger} c_{k_{x}^{\prime} s}$ in $\mathcal{H}_{\mathrm{HF}, P}^{(x)}$, i.e., intra-HES transitions are coupled to the nuclear spin operators perpendicular to the propagation direction. These differences again stem from the imaginary unit (or the lack thereof) in the spinors. Furthermore, the signs of $k_{x}$ and $k_{y}$ are again interchanged in the $\Lambda$ functions by comparing $\mathcal{H}_{\mathrm{HF}, P}^{(y)}$ and $\mathcal{H}_{\mathrm{HF}, P}^{(x)}$.

\section{Position averaged hyperfine interactions}

In HgTe, about $19 \%$ of the atoms have a nonzero nuclear spin and these can be assumed to be randomly distributed. In the HF interactions for the HESs, the unit-cell position of every nuclear spin is included. This information is sample dependent and often valuable insights can be found without it. Therefore we now consider the HF interactions averaged over the unit-cell position of the nuclear spins in analog to impurity averaging. ${ }^{97}$ To be specific, we focus here on the HF interactions (39) and (41) for the HESs along the $y$ axis. Mathematically, the position averaged of some quantity $\mathcal{F}$ is introduced as

$$
\overline{\mathcal{F}} \equiv \frac{1}{\mathcal{A}^{N_{s}}} \int_{\mathcal{A}} d \boldsymbol{\mathcal { R }}_{\diamond, 1} \cdots \int_{\mathcal{A}} d \boldsymbol{\mathcal { R }}_{\diamond, N_{s}} \mathcal{F}\left(\mathcal{R}_{1}, \ldots, \mathcal{R}_{N_{s}}\right)
$$

where $N_{s}$ is the number of nonzero nuclear spins covered by the HESs. We only average over the positions $\mathcal{R}_{\diamond, n} \equiv\left(\mathcal{X}_{n}, \mathcal{Z}_{n}\right)$ in the cross-section area $\mathcal{A}$ of the HESs along the $y$ axis. Thereby we keep the positions $\mathcal{Y}_{n}$, which break translational invariance along the edge and ultimately can lead to backscattering. ${ }^{61-64}$

Now we study the position averaged HF Hamiltonians. However, one can equally well position average at a later stage of a calculation, if it is physically relevant for a particular phenomenon, e.g., position averaging of the transition rates. ${ }^{61}$ Using the normalization of $f_{E \Gamma_{6}}$ in Appendix $\mathrm{B}$, the position averaged contact HF interaction (39) becomes

$$
\begin{aligned}
\overline{\mathcal{H}_{\mathrm{HF}, 1}^{(y)}}= & \frac{1}{4 \hbar} \frac{B-D}{2 B} \sum_{k_{y} k_{y}^{\prime}} \sum_{n} e^{i\left(k_{y}^{\prime}-k_{y}\right) \mathcal{Y}_{n}} \frac{A_{S, j_{n}}^{\text {Atomic }}}{N} \\
& \times\left(\overline{\Lambda_{k_{y}, k_{y}^{\prime}}} I_{z, n} c_{k_{y} u}^{\dagger} c_{k_{y}^{\prime} u}-\overline{\Lambda_{-k_{y},-k_{y}^{\prime}}} I_{z, n} c_{k_{y} d}^{\dagger} c_{k_{y}^{\prime} d}\right. \\
& \left.-\overline{\Lambda_{k_{y},-k_{y}^{\prime}}} I_{-, n} c_{k_{y} u}^{\dagger} c_{k_{y}^{\prime} d}-\overline{\Lambda_{-k_{y}, k_{y}^{\prime}}} I_{+, n} c_{k_{y} d}^{\dagger} c_{k_{y}^{\prime} u}\right),
\end{aligned}
$$

where the cross section area $\mathcal{A}$ is given in terms of the $\mathrm{QW}$ thickness $W_{z}$ and the HES width $W_{x}$ as $\mathcal{A}=W_{x} W_{z}$, such that $\int_{\mathcal{A}} d \boldsymbol{\mathcal { R }}_{\diamond, i}=1$. Here, $W_{x}$ is on the order of a few decay lengths $\lambda_{2}^{-1}$ and the number of atoms covered by the HESs is $N \equiv(L \mathcal{A}) / v_{a}$. We observe that the position dependent HF coupling $A_{S, j_{n}}\left(\mathcal{Z}_{n}\right)$ (17) is replaced by a homogenous $\mathrm{HF}$ coupling $A_{S, j_{n}}^{\text {Atomic }} / N$ due to the position averaging as in the case of quantum dots. ${ }^{98}$ The position average of the product of transverse functions, $\overline{\Lambda_{k, k^{\prime}}}=\int_{0}^{W_{x}} d \mathcal{X}_{n} g_{k}^{*}\left(\mathcal{X}_{n}\right) g_{k^{\prime}}\left(\mathcal{X}_{n}\right)$, is now independent of the positions $\mathcal{X}_{n}$. It can be well approximated by replacing $W_{x}$ by $\infty$ in the upper limit, which gives

$$
\begin{aligned}
\overline{\Lambda_{k_{y}, k_{y}^{\prime}}}= & \sqrt{\frac{2 \lambda_{1} \lambda_{2}\left(\lambda_{1}+\lambda_{2}\right)}{\left(\lambda_{1}-\lambda_{2}\right)^{2}} \sqrt{\frac{2 \lambda_{1}^{\prime} \lambda_{2}^{\prime}\left(\lambda_{1}^{\prime}+\lambda_{2}^{\prime}\right)}{\left(\lambda_{1}^{\prime}-\lambda_{2}^{\prime}\right)^{2}}}} \\
& \times\left(\frac{1}{\lambda_{1}^{\prime}+\lambda_{1}}-\frac{1}{\lambda_{2}^{\prime}+\lambda_{1}}-\frac{1}{\lambda_{1}^{\prime}+\lambda_{2}}+\frac{1}{\lambda_{2}^{\prime}+\lambda_{2}}\right),
\end{aligned}
$$

where $\lambda_{i}$ and $\lambda_{i}^{\prime}$ depends on $k_{y}$ and $k_{y}^{\prime}$, respectively. It is evident that $\overline{\Lambda_{k_{y}, k_{y}^{\prime}}}=\overline{\Lambda_{k_{y}^{\prime}, k_{y}}}$, since $g_{k_{y}}$ in Eq. (38a) is real. Moreover, $\overline{\Lambda_{k_{y}, k_{y}}}=1$ due to the normalization of $g_{k_{y}}$. Furthermore, in the particle-hole symmetric limit $D=0$, we have $g_{-k_{y}}(x)=g_{k_{y}}(x)$ such that ${\overline{\Lambda_{k_{y}, k_{y}^{\prime}}}}^{(D=0)}=$ ${\overline{\Lambda_{-k_{y},-k_{y}^{\prime}}}}^{(D=0)}={\overline{\Lambda_{k_{y},-k_{y}^{\prime}}}}^{(D=0)}={\overline{\Lambda_{-k_{y}, k_{y}^{\prime}}}}^{(D=0)}$. Hence the position averaged contact HF interaction (47) becomes isotropic in the particle-hole symmetric limit. Since the BHZ model is valid only close to the $\Gamma$ point, we expand $\overline{\Lambda_{k_{y}, k_{y}^{\prime}}}$ to lowest order in $k_{y}$ and $k_{y}^{\prime}$ for $D \neq 0$, i.e., $\overline{\Lambda_{k_{y}, k_{y}^{\prime}}} \simeq \eta_{k_{y}, k_{y}^{\prime}}$, where

$$
\eta_{k_{y}, k_{y}^{\prime}}=1-\frac{D^{2}\left[A^{2} B+2\left(B^{2}-D^{2}\right) M_{0}\right]}{8 B M_{0}^{2}\left(B^{2}-D^{2}\right)}\left(k_{y}-k_{y}^{\prime}\right)^{2} .
$$

Hence the lowest-order expansion fulfills $\eta_{k_{y}, k_{y}^{\prime}}=\eta_{-k_{y},-k_{y}^{\prime}}$ and $\eta_{-k_{y}, k_{y}^{\prime}}=\eta_{k_{y},-k_{y}^{\prime}}$ such that the position averaged contact HF interaction (47) simplifies to

$$
\begin{aligned}
\overline{\mathcal{H}_{\mathrm{HF}, 1}^{(y)}} \simeq & \frac{1}{4 \hbar} \frac{B-D}{2 B} \sum_{k_{y} k_{y}^{\prime}} \sum_{n} e^{i\left(k_{y}^{\prime}-k_{y}\right) \mathcal{Y}_{n}} \frac{A_{S, j_{n}}^{\text {Atomic }}}{N} \\
& \times\left[\eta_{k_{y}, k_{y}^{\prime}} I_{z, n}\left(c_{k_{y} u}^{\dagger} c_{k_{y}^{\prime} u}-c_{k_{y} d}^{\dagger} c_{k_{y}^{\prime} d}\right)\right. \\
& \left.-\eta_{k_{y},-k_{y}^{\prime}}\left(I_{-, n} c_{k_{y} u}^{\dagger} c_{k_{y}^{\prime} d}+I_{+, n} c_{k_{y} d}^{\dagger} c_{k_{y}^{\prime} u}\right)\right],
\end{aligned}
$$

to lowest order in $k_{y}$ and $k_{y}^{\prime}$. In this limit, $\overline{\mathcal{H}_{\mathrm{HF}, 1}^{(y)}}$ therefore has uniaxial anisotropy.

The position averaged HF interaction due to the $P$-like states in Eq. (41) becomes

$$
\begin{aligned}
\overline{\mathcal{H}_{\mathrm{HF}, P}^{(y)} \simeq} & \frac{2}{15 \hbar} \sum_{n} \sum_{k_{y}, k_{y}^{\prime}} e^{i\left(k_{y}^{\prime}-k_{y}\right) \mathcal{Y}_{n}} \frac{A_{P, j_{n}}^{\mathrm{Atomic}}}{N} \\
& \times\left[\eta_{k_{y}, k_{y}^{\prime}} \frac{7 B+5 D}{2 B} I_{z, n}\left(c_{k_{y} u}^{\dagger} c_{k_{y}^{\prime} u}-c_{k_{y} d}^{\dagger} c_{k_{y}^{\prime} d}\right)\right. \\
& \left.-\frac{B-D}{B} \eta_{k_{y},-k_{y}^{\prime}}\left(I_{-, n} c_{k_{y} u}^{\dagger} c_{k_{y}^{\prime} d}+I_{+, n} c_{k_{y} d}^{\dagger} c_{k_{y}^{\prime} u}\right)\right]
\end{aligned}
$$

by using the expansion $\overline{\Lambda_{k_{y}, k_{y}^{\prime}}} \simeq \eta_{k_{y}, k_{y}^{\prime}}$ and the normalization conditions for $f_{H}$ and $f_{E \Gamma_{8}}$ (see Appendix B). Interestingly, the terms in $\mathcal{H}_{\mathrm{HF}, P}^{(y)}(41)$ coupling $I_{x, n}$ and $c_{k_{y} \varsigma}^{\dagger} c_{k_{y}^{\prime} \varsigma}$ vanish in the position averaging, since $f_{H}(z)$ is even and $f_{E \Gamma_{8}}(z)$ is odd 25,76 such that $\overline{A_{P, j_{n}}^{E H}} \propto \int d \mathcal{Z}_{n} f_{E \Gamma_{8}}^{*}\left(\mathcal{Z}_{n}\right) f_{H}\left(\mathcal{Z}_{n}\right)=0$. Furthermore, even in the particle-hole symmetric limit $D=0, \overline{\mathcal{H}_{\mathrm{HF}, P}^{(y)}}$ is not isotropic in contrast to the contact HF interaction.

The total position averaged $\mathrm{HF}$ interaction $\overline{\mathcal{H}_{\mathrm{HF}}^{(y)}}=\overline{\mathcal{H}_{\mathrm{HF}, 1}^{(y)}}+\overline{\mathcal{H}_{\mathrm{HF}, P}^{(y)}}$ in the small wave-vector limit is 
TABLE II. Estimates of the effective HF couplings (53) for a pair of HESs. In parentheses, we give the percentage of the HF coupling stemming from the HF Hamiltonians due to $P$-like states, e.g., $4[(B-D) /(15 B)] A_{P, j_{n}}^{\text {Atomic }} / A_{j_{n}}^{\perp}$. Here, we use the atomic HF couplings in Table I and the BHZ model parameters ${ }^{1,94} B$ and $D$ only for a 70-Å-thick QW.

\begin{tabular}{lcccc}
\hline \hline & ${ }^{199} \mathrm{Hg}$ & ${ }^{201} \mathrm{Hg}$ & ${ }^{123} \mathrm{Te}$ & ${ }^{125} \mathrm{Te}$ \\
\hline$A_{j_{n}}^{z}(\mu \mathrm{eV})$ & 1.1 & -0.38 & -12 & -14 \\
& $(77 \%)$ & $(75 \%)$ & $(73 \%)$ & $(73 \%)$ \\
$A_{j_{n}}^{\perp}(\mu \mathrm{eV})$ & 0.30 & -0.11 & -3.5 & -4.2 \\
& $(14 \%)$ & $(12 \%)$ & $(12 \%)$ & $(12 \%)$ \\
\hline \hline
\end{tabular}

now found from Eqs. (50) and (51) to be

$$
\begin{aligned}
\overline{\mathcal{H}_{\mathrm{HF}}^{(y)}} \simeq & \frac{1}{2 \hbar} \sum_{n, k_{y} k_{y}^{\prime}} e^{i\left(k_{y}^{\prime}-k_{y}\right) \mathcal{Y}_{n}}\left[\frac{A_{j_{n}}^{z}}{N} \eta_{k_{y}, k_{y}^{\prime}} I_{z, n}\left(c_{k_{y} u}^{\dagger} c_{k_{y}^{\prime} u}-c_{k_{y} d}^{\dagger} c_{k_{y}^{\prime} d}\right)\right. \\
& \left.-\frac{A_{j_{n}}^{\perp}}{N} \eta_{k_{y},-k_{y}^{\prime}}\left(I_{-, n} c_{k_{y} u}^{\dagger} c_{k_{y}^{\prime} d}+I_{+, n} c_{k_{y} d}^{\dagger} c_{k_{y}^{\prime} u}\right)\right]
\end{aligned}
$$

where effective HF couplings were introduced as

$$
\begin{aligned}
A_{j_{n}}^{z} & \equiv \frac{B-D}{4 B} A_{S, j_{n}}^{\text {Atomic }}+\frac{4}{15} \frac{7 B+5 D}{2 B} A_{P, j_{n}}^{\text {Atomic }}, \\
A_{j_{n}}^{\perp} & \equiv \frac{B-D}{B}\left(\frac{1}{4} A_{S, j_{n}}^{\text {Atomic }}+\frac{4}{15} A_{P, j_{n}}^{\text {Atomic }}\right) .
\end{aligned}
$$

Hence the total position averaged $\mathrm{HF}$ interaction $\overline{\mathcal{H}_{\mathrm{HF}}^{(y)}}$ has uniaxial anisotropy. Estimates of $A_{j_{n}}^{z}$ and $A_{j_{n}}^{\perp}$ are given in Table II. Remarkably, the part of the effective HF couplings due to the $P$-like states dominates for the coupling $A_{j_{n}}^{z}$, but not for $A_{j_{n}}^{\perp}$. One reason is that the HESs have their main contribution on the $H$ states compared to the $E$ states, since $\left(\chi_{y}^{u}\right)^{T} \simeq(-i 0.36,-0.93,0,0)$ for a 70 - $\AA$-thick QW. ${ }^{94}$ Moreover, not only the $H$ states are $P$-like states, but also partly the $E$ states, see Eq. (5).

We remark that the position averaged HF interactions for the HESs along the $x$ axis Eqs. (44) and (45) follow along the same lines. The only difference in the total HF interaction in Eq. (52) is an opposite sign of the inter HES transition terms (apart from the replacements $\mathcal{Y}_{n} \rightarrow \mathcal{X}_{n}$ and $k_{y} \rightarrow k_{x}$ ). For typical parameters ${ }^{99}$ the number of atoms covered by the HESs is about $N \sim 10^{7}$ per $\mu \mathrm{m}$ edge, where about $19 \%$ of these atoms have a nonzero nuclear spin.

\section{DISCUSSION, SUMMARY, AND OUTLOOK}

In this paper, we have provided benchmark results within the BHZ model for the form and magnitude of (i) the contact HF interaction in Eq. (20), (ii) the dipole-dipole-like HF interaction in Eq. (31a), and (iii) the coupling of the electrons orbital momentum to the nuclear spin in Eq. (31b).

All the HF interactions couple the time-reversed blocks of the BHZ Hamiltonian (4b) - just as the Rashba spin-orbit coupling ${ }^{76}$ and the bulk inversion asymmetry terms. ${ }^{8}$ However, in contrast to the Rashba and bulk inversion asymmetry terms, the HF interactions break time-reversal symmetry from the electronic point of view. Therefore the HF interactions couple directly the Kramers pair of counterpropagating HESs of opposite wave numbers $(k$ and $-k)$, and thereby open for elastic backscattering. In contrast, the Rashba spin-orbit interaction combined with other scattering mechanisms can only couple the HESs inelastically. ${ }^{13,14,16,63}$ Hence our careful microscopic modeling of the HF interactions confirms that elastic backscattering spin-flip processes indeed are present as correctly anticipated on physical grounds in previous works on the interaction between HESs (modeled as spin-1/2) and one or more fixed magnetic moments. ${ }^{61-65}$

Furthermore, we estimated the atomic HF constants relevant for a $\mathrm{HgTe} \mathrm{QW}$, see Table I. These estimates are generally smaller by an order of magnitude or so compared to similar estimates for GaAs by Fischer et al. ${ }^{47,100}$ This is natural, since heavier elements often have lower HF couplings due to their higher principal quantum number of the outermost electron [see, e.g., Eqs. (D4) and (D5)]. As a consequence, the typical time for polarizing the nuclear spins by a current through the $\mathrm{HESs}^{61}$ of a HgTe QW is increased to hours or days compared to seconds for a GaAs QW in the quantum hall regime. ${ }^{54}$

From the HF Hamiltonians within the BHZ model, we derived a general formula (35) for the HF interactions for any nanostructure in a HgTe QW. The input of this formula is the envelope function of the given structure, where the effects of bulk inversion asymmetry, ${ }^{8}$ Rashba spin-orbit coupling, ${ }^{76}$ or magnetic fields ${ }^{21}$ can be included. From this formula, we found the HF interactions for a pair of HESs. Interestingly, the HF Hamiltonians depend on the orientation of the boundary at which the HESs propagate: the sign of the terms creating inter-HES transitions is opposite for perpendicular boundaries. This has not been considered previously in works on HESs coupled to fixed spins. ${ }^{61-65}$ On the level of transition rates between the HESs, ${ }^{61,62,64}$ such a difference is less important, since the rates are proportional to the HF matrix elements squared. However, this sign might play a role for more delicate phenomena such as Kondo physics ${ }^{63,65}$ or for HESs circulating one or more fixed spins.

We also found that the HF interactions due to the $P$-like states couple the intra-HES transitions to both nuclear spin components perpendicular to the propagation direction of the HESs, see Eqs. (41) and (45). The unusual terms coupling $I_{x, n}$ $\left(I_{y, n}\right)$ to the intra-HES transitions for propagation along the $y$ axis ( $x$ axis) were not included in previous studies. ${ }^{61-65}$ These terms might complicate the nature of nuclear spin polarization and its associated Overhauser effective magnetic field ${ }^{101}$ in a nontrivial way. For instance, this could affect the spin-orbit interaction induced backscattering processes between the HESs in the presence of a finite Overhauser field discussed in Ref. 64.

Finally, we averaged over the positions of the nuclear spins to remove the sample dependent information. This revealed that the total HF Hamiltonian is quite generally anisotropic and, moreover, that the contribution due to $P$-like states can dominate over the contact HF contribution, see Table II and Eq. (52). Therefore it can be important to include the HF interactions (1b) and (1c) relevant for $P$-like states for the HESs. Moreover, we found that the coupling of $I_{x, n}\left(I_{y, n}\right)$ to the intra-HES transitions for propagation along the $y$ axis ( $x$ axis) vanishes in the position averaging of the HF Hamiltonians. In this sense, these couplings are somewhat fragile compared to 
the usual coupling of $I_{z, n}$ to the intra-HES transitions. On the other hand, position averaging at a later stage of a calculation might allow interesting effects from these unusual terms to survive.

In passing, we remark that the nuclear spins can open a very small energy gap in the HES spectrum. This can be shown by averaging out all spacial directions of the nuclear spin positions in the total HF interaction. Treating the nuclear spins as a semiclassical field of zero mean value, ${ }^{102,103}$ the energy gap becomes proportional to the in-plane field. The ensemble averaged energy gap ${ }^{104}$ is proportional to $N^{-1 / 2}$ and estimated to be on the order of $10^{-4} \mu \mathrm{eV}$ for a micrometer-sized edge, which seems out of the current experimental range.

\section{ACKNOWLEDGMENTS}

We are especially grateful to Jan Fischer and Dietrich Rothe for helpful correspondence on their work in Refs. 47-50 and 76, respectively. We also thank Laurens Molenkamp, Björn Trauzettel, Andrzej Kdziorski, Jens Paaske, and Karsten Flensberg for useful discussions. Both AML and GP are supported by Grant No. MAT2011-24331 and by the ITN Grant 234970 (EU). AML acknowledges the Juan de la Cierva program (MICINN), Grant No. FIS2009-07277, and the Carlsberg Foundation. Furthermore, we acknowledge FIS2010-22438-E (Spanish National Network for Physics of Out-of-Equilibrium Systems).

\section{APPENDIX A: ON THE BHZ MODEL STATES}

This Appendix describes various details of the BHZ states $|E \pm\rangle$ and $|H \pm\rangle$. In particular, the time-reversal properties and the phase conventions of the envelope functions are discussed.

The states in the BHZ model as presented in Ref. 25 are given by

$$
\begin{aligned}
|E+\rangle & =f_{1}(z)\left|\Gamma_{6},+1 / 2\right\rangle+f_{4}(z)\left|\Gamma_{8},+1 / 2\right\rangle, \\
|H+\rangle & =f_{3}(z)\left|\Gamma_{8},+3 / 2\right\rangle, \\
|E-\rangle & =f_{2}(z)\left|\Gamma_{6},-1 / 2\right\rangle+f_{5}(z)\left|\Gamma_{8},-1 / 2\right\rangle, \\
|H-\rangle & =f_{6}(z)\left|\Gamma_{8},-3 / 2\right\rangle,
\end{aligned}
$$

similar to Eq. (5), but without specifying any phase conventions for the envelope functions $f_{n}(z)$. The lattice periodic functions can be given as

$$
\begin{aligned}
& \left|\Gamma_{6},+1 / 2\right\rangle=|S\rangle|\uparrow\rangle, \\
& \left|\Gamma_{6},-1 / 2\right\rangle=|S\rangle|\downarrow\rangle,
\end{aligned}
$$

and

$$
\begin{aligned}
\left|\Gamma_{8}, 3 / 2\right\rangle & =+\frac{1}{\sqrt{2}}\left[\left|P_{x}\right\rangle+i\left|P_{y}\right\rangle\right]|\uparrow\rangle, \\
\left|\Gamma_{8}, 1 / 2\right\rangle & =-\sqrt{\frac{2}{3}}\left|P_{z}\right\rangle|\uparrow\rangle+\frac{1}{\sqrt{6}}\left[\left|P_{x}\right\rangle+i\left|P_{y}\right\rangle\right]|\downarrow\rangle, \\
\left|\Gamma_{8},-1 / 2\right\rangle & =-\sqrt{\frac{2}{3}}\left|P_{z}\right\rangle|\downarrow\rangle-\frac{1}{\sqrt{6}}\left[\left|P_{x}\right\rangle-i\left|P_{y}\right\rangle\right]|\uparrow\rangle, \\
\left|\Gamma_{8},-3 / 2\right\rangle & =-\frac{1}{\sqrt{2}}\left[\left|P_{x}\right\rangle-i\left|P_{y}\right\rangle\right]|\downarrow\rangle,
\end{aligned}
$$

where the Bloch amplitudes $|S\rangle,\left|P_{x}\right\rangle,\left|P_{y}\right\rangle$, and $\left|P_{z}\right\rangle$ transform the same way as the well-known orbitals with the same names. ${ }^{75,105}$ The orbitals are connected to the spherical harmonics. ${ }^{73,96}$ Thus $\left|\Gamma_{8}, m_{j}\right\rangle$ correspond to $\mid j=3 / 2, m_{j}, l=$ $1, s=1 / 2\rangle$ in the angular momentum representation using the total angular momentum $\mathbf{J}=\mathbf{L}+\mathbf{S}$ as a good quantum number, where $\mathbf{S}$ is the electron spin in the basis $\{\uparrow, \downarrow\}$. Likewise, $\left|\Gamma_{6}, m_{j}\right\rangle$ simply corresponds to the $l=0$ state. Note that the split-off band $\Gamma_{7}$ with $j=1 / 2$ and $l=1$ is neglected in the BHZ model. Here, $|S\rangle$ is chosen to be purely imaginary ${ }^{106}$ and $\left|P_{x}\right\rangle,\left|P_{y}\right\rangle$, and $\left|P_{z}\right\rangle$ to be real. ${ }^{73}$ Furthermore, we follow the convention by Bernevig et al. ${ }^{25}$ and Novik et al.$^{107}$ by using an overall opposite $\operatorname{sign}^{108}$ for the $\Gamma_{8}$ states in terms of the $P$ states in Eq. (A3) compared to other authors. ${ }^{73,109}$ This sign change is not important for the purposes of this paper.

Next we discuss the phase conventions for the envelope functions made in the main text. The envelope functions $f_{n}(z)(n=1, \ldots, 6)$ are found from the Luttinger-Kane model at $k_{x}=k_{y}=0$ and therefore has to fulfill the following differential equations: ${ }^{25,76}$

$$
\begin{gathered}
T f_{n}(z)-\sqrt{\frac{2}{3}} P_{0} i \partial_{z} f_{n+3}(z)=E_{\mathbf{k}=0} f_{n}(z), \\
-\sqrt{\frac{2}{3}} P_{0} i \partial_{z} f_{n}(z)+W_{-} f_{n+3}(z)=E_{\mathbf{k}=0} f_{n+3}(z),
\end{gathered}
$$

for $n=1,2$ only [i.e., only for the two pairs $\left(f_{1}, f_{4}\right)$ and $\left.\left(f_{2}, f_{5}\right)\right]$. Similarly, ${ }^{25,76}$

$$
W_{+} f_{n}(z)=E_{\mathbf{k}=0} f_{n}(z), \quad \text { for } n=3,6 \text { only. }
$$

Here, we have introduced the real operators

$$
\begin{aligned}
T & =E_{c}(z)+\frac{\hbar^{2}}{2 m_{e}} k_{z}[2 F(z)+1] k_{z}, \\
W_{ \pm} & =E_{v}(z)+\frac{\hbar^{2}}{2 m_{e}} k_{z}\left[2 \gamma_{2}(z) \mp \gamma_{1}(z)\right] k_{z},
\end{aligned}
$$

where $k_{z}=-i \partial_{z}, E_{c(v)}$ is the conduction (valence) band edge, $m_{e}$ the bare electron mass, $\gamma_{1,2}$ are the Luttinger parameters, ${ }^{110}$ and $F(z)$ is a real function including the remote $\Gamma_{5}$ bands perturbatively. ${ }^{76}$ The parameters $\gamma_{1,2}, F$, and $E_{c, v}$ are different in the HgTe and CdTe layers of the heterostructure, which leads to the $z$ dependence. The solution of these equations will also give the energy for that particular solution (energy band) $E_{\mathbf{k}=0}$ at $\mathbf{k}=(0,0)$. From Eq. (A5), it follows that we can choose $f_{3}(z)=f_{6}(z)$, which is simply denoted as $f_{H}(z)$ in the main text. Furthermore, Eq. (A4) allows us to choose $f_{1}(z)=$ $f_{2}(z)$ and $f_{4}(z)=f_{5}(z)$, which are called $f_{E \Gamma_{6}}(z)$ and $f_{E \Gamma_{8}}(z)$, respectively, in the main text. By comparison of Eq. (A4) and their complex conjugates, it follows that we can choose $f_{1}(z)$ real and $f_{4}(z)$ purely imaginary as in Ref. 76 .

Now we turn our attention to the time-reversal properties of the states $|E \pm\rangle$ and $|H \pm\rangle$. The time-reversal operator $\Theta$ is defined up to an arbitrary phase factor. Here, we use $\Theta=-i \sigma_{y} K$, where $K$ is the complex conjugation operator and $\sigma_{y}$ a Pauli matrix in electron spin space. The time-reversal operator $\Theta$ acts differently in different bases (due to the complex conjugation), so one should stick to the same basis through out a calculation. ${ }^{96}$ The $\left|\Gamma_{i}, m_{j}\right\rangle$ states under the time-reversal operator follow from Eqs. (A2) and (A3) by using that the $P$-like states are real, the $S$-like states are pure 
imaginary and that $\Theta|\uparrow\rangle=+|\downarrow\rangle$ and $\Theta|\downarrow\rangle=-|\uparrow\rangle$, i.e.,

$$
\begin{aligned}
& \Theta\left|\Gamma_{6}, \pm 1 / 2\right\rangle=\mp\left|\Gamma_{6}, \mp 1 / 2\right\rangle, \\
& \Theta\left|\Gamma_{8}, \pm 1 / 2\right\rangle= \pm\left|\Gamma_{8}, \mp 1 / 2\right\rangle, \\
& \Theta\left|\Gamma_{8}, \pm 3 / 2\right\rangle=\mp\left|\Gamma_{8}, \mp 3 / 2\right\rangle .
\end{aligned}
$$

Therefore we can now evaluate, e.g., $\Theta|E+\rangle$ by using Eq. (A7) and that $f_{E \Gamma_{6}}$ is real and $f_{E \Gamma_{8}}$ is purely imaginary, which gives $\Theta|E+\rangle=-|E-\rangle$. Hence our conventions lead to

$$
\begin{aligned}
\Theta|E \pm\rangle & =\mp|E \mp\rangle, \\
\Theta|H \pm\rangle & =\mp|H \mp\rangle,
\end{aligned}
$$

which fulfill $\Theta^{2}=-1$ as expected. We remark that that Rothe et al. ${ }^{76}$ find opposite signs under time-reversal (i.e., $\Theta|E \pm\rangle=$ $\pm|E \mp\rangle$ and $\Theta|H \pm\rangle= \pm|H \mp\rangle$ ), simply because an opposite overall sign was chosen in the definition of the time-reversal operator. ${ }^{111}$ In Ref. 67, the same signs as in Eq. (A8) are found.

\section{APPENDIX B: NORMALIZATION OF THE BHZ STATES}

In this Appendix, the normalization of the envelope functions and lattice periodic functions within the envelope function approximation is discussed. To this end, we use $\varphi_{\mathbf{k}, H+}(\mathbf{r})$ in Eq. (7b) as an example. The entire wave function is normalized in the usual way, i.e.,

$$
\int_{\mathcal{V}} d \mathbf{r}\left|\varphi_{\mathbf{k}, H+}(\mathbf{r})\right|^{2}=1
$$

where $\mathcal{V}$ is the volume of the entire system. The normalization of the entire wave function (B1) leaves a freedom to normalize the envelope function and the lattice periodic function in the most convenient way for the problem at hand. Various choices are found in the literature, see, e.g., footnote 2 in the review of Coish and Baugh. ${ }^{30}$

To see how this normalization choice works in practice, we begin by separating the left-hand side of the normalization condition (B1) into a product of the envelope function and the lattice periodic function normalization, respectively. To this end, the normalization condition (B1) is rewriting by dividing the integral over the entire space into a sum of integrals over the unit cells as in Eq. (9), i.e.,

$$
\begin{aligned}
1 & =\frac{v_{a}}{L_{x} L_{y}} \int_{\mathcal{V}} d \mathbf{r}\left|f_{H}(z)\right|^{2}\left|u_{\Gamma_{8},+\frac{3}{2}}(\mathbf{r})\right|^{2} \\
& =\frac{v_{a}}{L_{x} L_{y}} \sum_{\mathcal{R}_{n}} \int_{v_{u c}^{(n)}} d \boldsymbol{\rho}\left|f_{H}\left(\rho_{z}+\mathcal{Z}_{n}\right)\right|^{2}\left|u_{\Gamma_{8},+\frac{3}{2}}\left(\mathcal{R}_{n}+\boldsymbol{\rho}\right)\right|^{2} \\
& \simeq \frac{v_{a}}{L_{x} L_{y}} \sum_{\mathcal{R}_{n}} \int_{v_{u c}^{(n)}} d \boldsymbol{\rho}\left|f_{H}\left(\mathcal{Z}_{n}\right)\right|^{2}\left|u_{\Gamma_{8},+\frac{3}{2}}(\boldsymbol{\rho})\right|^{2} \\
& =\frac{v_{a}}{L_{x} L_{y}}\left[\sum_{\mathcal{R}_{n}}\left|f_{H}\left(\mathcal{Z}_{n}\right)\right|^{2}\right]\left[\int_{v_{u c}} d \boldsymbol{\rho}\left|u_{\Gamma_{8},+\frac{3}{2}}(\boldsymbol{\rho})\right|^{2}\right],
\end{aligned}
$$

where we used in the third equality that the envelope function - by construction - is slowly varying on the scale of the unit cell, so $f_{H}\left(\rho_{z}+\mathcal{Z}_{n}\right) \simeq f_{H}\left(\mathcal{Z}_{n}\right)$, and that the lattice periodic functions are periodic with the lattice, i.e.,
$u_{\Gamma_{8},+\frac{3}{2}}\left(\mathcal{R}_{n}+\boldsymbol{\rho}\right)=u_{\Gamma_{8},+\frac{3}{2}}(\boldsymbol{\rho})$ for all lattice vectors $\boldsymbol{\mathcal { R }}_{n}$. The integral of $\left|u_{\Gamma_{8},+\frac{3}{2}}(\boldsymbol{\rho})\right|^{2}$ over the $n$th unit cell is the same for every unit cell and hence independent of $n$, which we indicate by $v_{u c}^{(n)} \rightarrow v_{u c}$. Moreover, the sum over lattice points in Eq. (B2) can be made into an integral (including the unit cell volume $v_{u c}$ ), since the envelope function varies slowly on the interatomic scale, i.e., $\sum_{\mathcal{R}_{n}}\left|f_{H}\left(\mathcal{Z}_{n}\right)\right|^{2} \simeq \frac{1}{v_{u c}} \int_{\mathcal{V}} d \mathcal{R}\left|f_{H}(\mathcal{Z})\right|^{2}$. Therefore we arrive at

$$
1=\frac{v_{a}}{v_{u c}}\left[\frac{1}{L_{x} L_{y}} \int_{\mathcal{V}} d \mathcal{R}\left|f_{H}(\mathcal{Z})\right|^{2}\right]\left[\int_{v_{u c}} d \boldsymbol{\rho}\left|u_{\Gamma_{8},+\frac{3}{2}}(\boldsymbol{\rho})\right|^{2}\right],
$$

where the normalization of the entire wave function in Eq. (B1) have been written as a product of the normalization of the envelope function and lattice periodic function part, respectively. Thus it is now clear that some freedom exists in the normalization choice.

In this paper, we normalize the lattice periodic function as Fischer et al., ${ }^{4-50}$ i.e.,

$$
\int_{v_{u c}} d \rho\left|u_{\Gamma_{8},+\frac{3}{2}}(\rho)\right|^{2}=\frac{v_{u c}}{v_{a}}=2
$$

using the fact that a zinc-blende crystal, like HgTe or GaAs, contains two atoms per unit cell, $v_{u c}=2 v_{a}$. This normalization has the advantage that the atomic HF constants found in the main paper are independent of the number of atoms in the unit cell. ${ }^{30}$ Moreover, the envelope function is normalized as

$$
\frac{1}{L_{x} L_{y}} \int_{\mathcal{V}} d \mathcal{R}\left|f_{H}(\mathcal{Z})\right|^{2}=1
$$

such that Eq. (B3) is fulfilled.

The normalization procedure follows the same lines as above for the other BHZ basis functions, e.g., all lattice periodic functions are normalized to the number of atoms in the unit cell. When the wave function is not a simple product of an envelope function and a lattice periodic function, then it should be used that different lattice periodic functions are orthogonal, i.e., $\int_{v_{u c}} d \rho u_{\Gamma_{a}, m_{j}}^{*}(\rho) u_{\Gamma_{b}, m_{j}^{\prime}}(\rho)=2 \delta_{\Gamma_{a}, \Gamma_{b}} \delta_{m_{j}, m_{j}^{\prime}}$. Finally, it should be noted that for the $E \pm$ states, we end up with a combined normalization for the two envelope functions, i.e. $\int d z\left[\left|f_{E \Gamma_{6}}(z)\right|^{2}+\left|f_{E \Gamma_{8}}(z)\right|^{2}\right]=1$. We assume that each of these two envelope functions are normalized to one half. ${ }^{25}$

\section{APPENDIX C: DETAILS ON THE CALCULATION OF THE ATOMIC INTEGRALS OF THE HYPERFINE INTERACTIONS FOR $P$-LIKE STATES}

This Appendix deals with the integrals over the atomic wave functions of the form

$$
\int_{v_{u c}} d \rho\left[\Psi_{\Gamma_{8}, m_{j}}^{\mathrm{Hg} / \mathrm{Te}}(\rho \mp \mathbf{d} / 2)\right]^{*} h_{i}^{n} \Psi_{\Gamma_{8}, m_{j}^{\prime}}^{\mathrm{Hg} / \mathrm{Te}}(\rho \mp \mathbf{d} / 2),
$$

which appear in the matrix elements of $H_{\mathrm{HF}, 2}$ and $H_{\mathrm{HF}, 3}$ in Sec. IVC. The atomic wave functions are written as $\Psi_{\Gamma_{8}, m_{j}}^{\mathrm{Hg} / \mathrm{Te}}(\mathbf{r})=R^{\mathrm{Hg} / \mathrm{Te}}(r) \mathbb{Y}_{\Gamma_{8}, m_{j}}(\theta, \phi)$, i.e., a product of a radial and an angular part as in the main text. The angular part 
of the wave functions $\mathbb{Y}_{\Gamma_{8}, m_{j}}(\theta, \phi)$ are combinations of the usual spherical harmonics ${ }^{96} Y_{l}^{m}(\theta, \phi)$ and the electronic spin-1/2 (| $\rangle$ and $|\downarrow\rangle)$ and inherit the symmetry of the bands, ${ }^{47,84}$ i.e.,

$$
\begin{aligned}
& \mathbb{Y}_{\Gamma_{8},+\frac{3}{2}}(\theta, \phi)=-Y_{1}^{1}(\theta, \phi)|\uparrow\rangle, \\
& \mathbb{Y}_{\Gamma_{8},+\frac{1}{2}}(\theta, \phi)=-\sqrt{\frac{2}{3}} Y_{1}^{0}(\theta, \phi)|\uparrow\rangle-\sqrt{\frac{1}{3}} Y_{1}^{1}(\theta, \phi)|\downarrow\rangle, \\
& \mathbb{Y}_{\Gamma_{8},-\frac{1}{2}}(\theta, \phi)=-\sqrt{\frac{2}{3}} Y_{1}^{0}(\theta, \phi)|\downarrow\rangle-\sqrt{\frac{1}{3}} Y_{1}^{-1}(\theta, \phi)|\uparrow\rangle, \\
& \mathbb{Y}_{\Gamma_{8},-\frac{3}{2}}(\theta, \phi)=-Y_{1}^{-1}(\theta, \phi)|\downarrow\rangle,
\end{aligned}
$$

which are all eigenfunctions of $J_{z}=L_{z}+S_{z}$ (with eigenvalue $\hbar m_{j}$ ), $\mathbf{J}^{2}=(\mathbf{L}+\mathbf{S})^{2}$ (with $j=3 / 2$ ), $\mathbf{L}^{2}$ (with $l=1$ due to $P$ states), and $\mathbf{S}^{2}$ (with $s=1 / 2$ ). To be consistent with the BHZ model, we use the same overall sign as Refs. 25 and 107 , which is opposite to the one used in, e.g., Refs. 73 and 109 [see also Appendix A, Eq. (A3), and endnote 108]. However, this overall sign cancels out in the matrix elements between $P$ states and therefore has no effect here.

To find the integrals ( $\mathrm{C} 1)$, the spherical approximation Eq. (24) is used. This is an excellent approximation, since most of the weight of the integrals are close to the atomic core. To facilitate the calculations, the HF dipole-dipolelike interaction for a single nuclear spin $h_{2}^{n}$ Eq. (1b) is rewritten as (choosing the origin at the nuclear spin, i.e., $\mathbf{r}_{n}=$ $\mathbf{r}-\mathbf{R}_{n} \rightarrow \mathbf{r}$ )

$$
\begin{aligned}
h_{2}^{n}= & \frac{\mu_{0}}{4 \pi} \gamma_{e} \gamma_{j_{n}} \frac{1}{r^{3}\left(1+\frac{r_{c}}{r}\right)} \frac{1}{2} \\
& \times\left\{\left(\frac{3 z^{2}-r^{2}}{r^{2}}\right)\left[2 S_{z} I_{z, n}-\frac{1}{2}\left(S_{+} I_{-, n}+S_{-} I_{+, n}\right)\right]\right. \\
& +3\left(\frac{x^{2}-y^{2}}{r^{2}}\right) \frac{1}{2}\left(S_{+} I_{+, n}+S_{-} I_{-, n}\right) \\
& +\frac{6 x y}{r^{2}}\left(S_{x} I_{y, n}+S_{y} I_{x, n}\right)+\frac{6 x z}{r^{2}}\left(S_{x} I_{z, n}+S_{z} I_{x, n}\right) \\
& \left.+\frac{6 y z}{r^{2}}\left(S_{y} I_{z, n}+S_{z} I_{y, n}\right)\right\} .
\end{aligned}
$$

This is written in such a way that the integrals [like Eq. (24)] consist of a radial integral over $\propto \frac{1}{r^{3}\left(1+\frac{r_{c}}{r}\right)}$ times a sum of angular integrals. The terms in the curly bracket become the sum of angular integrals, where the space dependencies are seen to form spherical tensor operators or sums thereof. Therefore the Wigner-Eckart theorem is useful to identify the integrals that are zero, see, e.g., Ref. 96. As an example, the element for a $\mathrm{Hg}$ nuclear spin between the $\Psi_{\Gamma_{8}, \tau 3 / 2}^{\mathrm{Hg}}(\mathbf{r})$ states, appearing in the matrix element $\left\langle\varphi_{\mathbf{k} H \tau}\left|H_{\mathrm{HF}, 2}\right| \varphi_{\mathbf{k}^{\prime} H \tau^{\prime}}\right\rangle$, is found to be (after some calculations)

$$
\begin{gathered}
\int_{0}^{r_{\max }} d r r^{2} \int_{0}^{2 \pi} d \phi \int_{0}^{\pi} d \theta \sin (\theta)\left[\Psi_{\Gamma_{8}, \tau 3 / 2}^{\mathrm{Hg}}(\mathbf{r})\right]^{*} h_{2}^{n} \Psi_{\Gamma_{8}, \tau^{\prime} 3 / 2}^{\mathrm{Hg}}(\mathbf{r}) \\
=\frac{\mu_{0}}{4 \pi} \gamma_{e} \gamma_{j_{n}}\left(\frac{1}{r^{3}}\right)_{r}^{\mathrm{Hg}} \delta_{\tau, \tau^{\prime}}\left(-\frac{1}{5} \tau \hbar I_{z, n}\right)
\end{gathered}
$$

using Eqs. (C2) and (C3) and the definition (25). The rest of the integrals for $h_{2}^{n}$ are found similarly.
Finally, we note that the integrals (C1) involving $h_{3}^{n}(1 \mathrm{c})$ are much simpler to evaluate. The integrals in the spherical approximation (24) again separate into the radial integral $\left\langle 1 / r^{3}\right\rangle_{r}^{\mathrm{Hg} / \mathrm{Te}}$ times a sum of angular integrals, which can be found by using the rewriting $\mathbf{L}_{n} \cdot \mathbf{I}_{n}=L_{z, n} I_{z, n}+\frac{1}{2}\left(L_{+, n} I_{-, n}+L_{-, n} I_{+, n}\right)$ and Eq. (C2).

\section{APPENDIX D: ESTIMATION OF THE ATOMIC HF CONSTANTS}

In this Appendix, we estimate the atomic HF couplings Eqs. (18) and (26),

$$
\begin{aligned}
& A_{S, j_{n}}^{\text {Atomic }}=\frac{2 \mu_{0}}{3} g_{e} \mu_{B} g_{j_{n}} \mu_{N}\left|u_{\Gamma_{6}}\left(\mathbf{R}_{n}\right)\right|^{2}, \\
& A_{P, j_{n}}^{\text {Atomic }}=\frac{\mu_{0}}{4 \pi} g_{e} \mu_{B} g_{j_{n}} \mu_{N}\left(N_{\Gamma_{8}}\right)^{2}\left|\alpha_{j_{n}}\right|^{2}\left\langle\frac{1}{r^{3}}\right\rangle_{r}^{j_{n}}
\end{aligned}
$$

along the same lines as Fischer et $a l .{ }^{47}$ These estimates are given in Table I. Below, we go through the ingredients to make these estimates.

Within the LCAO approach (13), the lattice periodic functions within a unit cell are written as a linear combination of the two atomic orbitals. The relative weight between the two orbitals is related to the ionicity and found to be $\mathrm{b}^{112,113}$

$$
\alpha_{\mathrm{Te}} \simeq \sqrt{0.8} \text { and } \alpha_{\mathrm{Hg}} \simeq \sqrt{0.2},
$$

which is taken to be the same for the $\Gamma_{6}$ and $\Gamma_{8}$ bands. ${ }^{47}$

Moreover, the $g$ factors for the various isotopes are ${ }^{31}$

$$
\begin{aligned}
& g^{199} \mathrm{Hg}=1.01, \quad g^{201} \mathrm{Hg}=-0.37, \\
& g_{123} \mathrm{Te}=-1.47, \quad g^{125} \mathrm{Te}=-1.78 .
\end{aligned}
$$

These are seen to vary in sign, which is the reason for the sign variation of the HF couplings.

Furthermore, to estimate the HF couplings, the atomic wave functions $\Psi_{\Gamma_{i}, m_{j}}^{\mathrm{Hg} / \mathrm{Te}}$ also have to be given explicitly. The angular part follows the band symmetry as in Eq. (C2). As for the radial part, we follow Fischer et al. ${ }^{47}$ and approximate it by a hydrogenic radial eigenfunction ${ }^{96} R_{n l}(r)$ with an effective charge $e Z_{\text {eff }}$ replacing the actual charge of the nucleus $e Z$ in order to include atomic screening effects, etc., i.e., $Z_{\text {eff }}<Z$. The outermost electrons in $\mathrm{Hg}(\mathrm{Te})$ have the principal quantum number $n=6(n=5)$ such that $R_{\Gamma_{8}}^{\mathrm{Hg}(\mathrm{Te})}(r)=R_{6(5), 1}(r)$ and $R_{\Gamma_{6}}^{\mathrm{Hg}(\mathrm{Te})}(r)=R_{6(5), 0}(r)$. Clementi et al. ${ }^{114,115}$ have calculated the effective charges $Z_{\text {eff }}$ for various atoms and orbitals and found that $Z_{\mathrm{eff}}(\mathrm{Te}, 5 s)=12.5, Z_{\mathrm{eff}}(\mathrm{Te}, 5 p)=10.8$ and $Z_{\text {eff }}(\mathrm{Hg}, 6 s / 6 p)=11.2$, which obviously is much smaller than the bare nuclear charges $e Z=52 e$ for $\mathrm{Te}$ and $e Z=80 e$ for $\mathrm{Hg}$.

Using $\left|u_{\Gamma_{6}}\left(\mathbf{R}_{n}\right)\right|^{2} \simeq\left(N_{\Gamma_{6}, 1 / 2}\right)^{2}\left|\alpha_{j}\right|^{2}\left|\Psi_{\Gamma_{6}}^{j}(\mathbf{0})\right|^{2}$ with the hydrogenic orbital $\Psi_{\Gamma_{6}}^{j}(\mathbf{r})=R_{n 0}(r) Y_{0}^{0}(\theta, \phi)$ for isotope $j$, we can now give the atomic contact $\mathrm{HF}$ coupling as

$$
A_{S, j}^{\text {Atomic }} \simeq \frac{2 \mu_{0}}{3} g_{e} \mu_{B} g_{j} \mu_{N}\left[N_{\Gamma_{6}, 1 / 2}\right]^{2}\left|\alpha_{j}\right|^{2} \frac{\left[Z_{\mathrm{eff}}(j, s)\right]^{3}}{\pi a_{0}^{3} n^{3}},
$$

where $a_{0}=4 \pi \epsilon_{0} \hbar^{2} /\left(m_{e} e^{2}\right)$ is the Bohr radius and $\epsilon_{0}$ is permittivity of free space. 
The hydrogeniclike atomic orbitals also makes it easy to calculate $\left\langle 1 / r^{3}\right\rangle_{r}^{j}$ in Eq. (25) numerically, which shows that neither the nuclear length scale $r_{c}$ nor $r_{\max }$ make a difference in practice. Hence we can use

$$
\left\langle\frac{1}{r^{3}}\right\rangle_{r}^{j} \simeq \int_{0}^{\infty} d r r^{2}\left|R_{n, l=1}(r)\right|^{2} \frac{1}{r^{3}}=\frac{\left[Z_{\mathrm{eff}}(j, p)\right]^{3}}{3 a_{0}^{3} n^{3}},
$$

to find the HF coupling $A_{P, j}^{\text {Atomic }}$ for the $P$ states.

Therefore now we only need one more ingredient to be able to estimate the HF couplings, namely the normalization constants $N_{\Gamma_{i}, m_{j}}$ of the lattice periodic functions in the LCAO approach Eq. (13). The normalization condition (B4) leads to

$$
\int_{v_{u c}} d \mathbf{r}\left|\alpha_{\mathrm{Te}} \Psi_{\Gamma_{i}, m_{j}}^{\mathrm{Te}}\left(\mathbf{r}+\frac{\mathbf{d}}{2}\right) \pm \alpha_{\mathrm{Hg}} \Psi_{\Gamma_{i}, m_{j}}^{\mathrm{Hg}}\left(\mathbf{r}-\frac{\mathbf{d}}{2}\right)\right|^{2}=\frac{2}{N_{\Gamma_{i}, m_{j}}^{2}},
$$

where $+(-)$ corresponds to $\Gamma_{8}\left(\Gamma_{6}\right)$. First of all, we note that

$$
N_{\Gamma_{i}, m_{j}}=N_{\Gamma_{i},-m_{j}}
$$

due to the similar form of the atomic wave functions for $\pm m_{j}$, see, e.g., Eq. (C2). Using the hydrogenic eigenstates, we can therefore now numerically find the normalization constants $N_{\Gamma_{i}, m_{j}}$. Numerically, these do depend weakly on how the Wigner-Seitz unit cell of the zinc-blende crystal is approximated, in contrast to the unit cell integrals involving $h_{i}^{n}$ for $i=2,3$ in Sec. IV C. We have tested various spherical and cubic approximations to the primitive Wigner-Seitz unit cell all with the same volume as the Wigner-Seitz unit cell, namely $v_{u c}=16|\mathbf{d}|^{3} /(3 \sqrt{3})$, where $|\mathbf{d}|=0.279 \mathrm{~nm}$ is the distance between the $\mathrm{Hg}$ and $\mathrm{Te}$ atoms in unit cell, see, e.g., p. 58 in Ref. 33. Such a weak dependence is also found in the estimate for GaAs by Fischer et al. ${ }^{47}$ From our various approximate unit cell calculation, we found that a good estimate for the normalization constants are $\left(N_{\Gamma_{8}, 1 / 2}\right)^{2} \simeq$ $\left(N_{\Gamma_{8}, 3 / 2}\right)^{2} \simeq 3.6$ and $\left(N_{\Gamma_{6}, 1 / 2}\right)^{2} \simeq 2.7$. Therefore we can use approximately equal normalization constants for $u_{\Gamma_{8}, \pm 1 / 2}$ and $u_{\Gamma_{8}, \pm 3 / 2}$, which allows for the introduction of a common atomic $P$-like HF constant in Eq. (26). Therefore we now have all the ingredients to make the estimates with the results seen in Table I.

\section{APPENDIX E: HYPERFINE INTERACTIONS IN TERMS OF EDGE STATE SPIN OPERATORS}

Here, we reformulate the HF interactions (39) and (41) for the HESs along the $y$ axis in order to give some more insights into their form. Having in mind the spin-1/2 picture of a pair of HESs discussed in Sec. VI A, we are lead to introduce the nondiagonal edge states spin operators as

$$
\begin{aligned}
\mathfrak{s}_{k_{y} k_{y}^{\prime}, x} & =\frac{\hbar}{2}\left(c_{k_{y} u}^{\dagger} c_{k_{y}^{\prime} d}+c_{k_{y} d}^{\dagger} c_{k_{y}^{\prime} u}\right), \\
\mathfrak{s}_{k_{y} k_{y}^{\prime}, y} & =i \frac{\hbar}{2}\left(c_{k_{y} d}^{\dagger} c_{k_{y}^{\prime} u}-c_{k_{y} u}^{\dagger} c_{k_{y}^{\prime} d}\right), \\
\mathfrak{s}_{k_{y} k_{y}^{\prime}, z} & =\frac{\hbar}{2}\left(c_{k_{y} u}^{\dagger} c_{k_{y}^{\prime} u}-c_{k_{y} d}^{\dagger} c_{k_{y}^{\prime} d}\right),
\end{aligned}
$$

together with the operator $\mathfrak{I}_{k_{y} k_{y}^{\prime}}=\frac{\hbar}{2}\left(c_{k_{y} u}^{\dagger} c_{k_{y}^{\prime} u}+c_{k_{y} d}^{\dagger} c_{k_{y}^{\prime} d}\right)$ and the raising and lowering operators $\mathfrak{s}_{k_{y} k_{y}^{\prime}, \pm} \equiv \mathfrak{s}_{k_{y} k_{y}^{\prime}, x} \pm i \mathfrak{s}_{k_{y} k_{y}^{\prime}, y}$ for the edge state spin. Here, for instance, $\mathfrak{s}_{k_{y} k_{y}^{\prime},+}$ moves a particle in the state $\Psi_{y, k_{y}^{\prime}}^{d}$ into the state $\Psi_{y, k_{y}}^{u}$ and in this sense raises the edge state spin (while also changing the wave vector). In the case of $k_{y}=k_{y}^{\prime}$, the edge state spin operators (E1) coincide with the usual spin- $1 / 2$ operators ${ }^{97}$ and $\mathfrak{I}_{k_{y} k_{y}}$ is the particle number operator (times $\hbar / 2$ ).

The contact HF interaction (39) in terms of the edge state spin operators (E1) becomes

$$
\begin{aligned}
\mathcal{H}_{\mathrm{HF}, 1}^{(y)}= & \frac{1}{2 \hbar^{2}} \frac{B-D}{2 B} \sum_{k_{y} k_{y}^{\prime}} \sum_{n} \frac{e^{i\left(k_{y}^{\prime}-k_{y}\right) \mathcal{Y}_{n}}}{L_{y}} A_{S, j_{n}}\left(\mathcal{Z}_{n}\right) \\
& \times\left[\left(\Lambda_{k_{y}, k_{y}^{\prime}}^{\left(\mathcal{X}_{n}\right)}+\Lambda_{-k_{y},-k_{y}^{\prime}}^{\left(\mathcal{X}_{n}\right)}\right) I_{z, n} \mathfrak{s}_{k_{y} k_{y}^{\prime}, z}\right. \\
& +\left(\Lambda_{k_{y}, k_{y}^{\prime}}^{\left(\mathcal{X}_{n}\right)}-\Lambda_{-k_{y},-k_{y}^{\prime}}^{\left(\mathcal{X}_{n}\right)}\right) I_{z, n} \mathfrak{I}_{k_{y} k_{y}^{\prime}} \\
& \left.-\Lambda_{k_{y},-k_{y}^{\prime}}^{\left(\mathcal{X}_{n}\right)} I_{-, n} \mathfrak{s}_{k_{y} k_{y}^{\prime},+}-\Lambda_{-k_{y}, k_{y}^{\prime}}^{\left(\mathcal{X}_{y}\right)} I_{+, n} \mathfrak{s}_{k_{y} k_{y}^{\prime},-}\right] .
\end{aligned}
$$

Using the edge state spin operators (E1), the HF interaction (41) due to the $P$-like states becomes

$$
\begin{aligned}
\mathcal{H}_{\mathrm{HF}, P}^{(y)}= & \frac{2}{15 \hbar^{2}} \sum_{n} \sum_{k_{y}, k_{y}^{\prime}} \frac{e^{i\left(k_{y}^{\prime}-k_{y}\right)}}{L_{y}}\left[-2 \frac{B-D}{B}\right. \\
& \times A_{P, j_{n}}^{E E}\left(\Lambda_{k_{y},-k_{y}^{\prime}}^{\left(\mathcal{X}_{n}\right)} I_{-, n} \mathfrak{s}_{k_{y} k_{y}^{\prime},+}+\Lambda_{-k_{y}, k_{y}^{\prime}}^{\left(\mathcal{X}_{n}\right)} I_{+, n} \mathfrak{s}_{k_{y} k_{y}^{\prime},-}\right) \\
& +\left(\Lambda_{k_{y}, k_{y}^{\prime}}^{\left(\mathcal{X}_{n}\right)}+\Lambda_{-k_{y},-k_{y}^{\prime}}^{\left(\mathcal{X}_{n}\right)}\right)\left(\mathfrak{L}_{n} I_{x, n}+\mathfrak{F}_{n} I_{z, n}\right) \mathfrak{s}_{k_{y} k_{y}^{\prime}, z} \\
& \left.+\left(\Lambda_{k_{y}, k_{y}^{\prime}}^{\left(\mathcal{X}_{n}\right)}-\Lambda_{-k_{y},-k_{y}^{\prime}}^{\left(\mathcal{X}_{n}\right)}\right)\left(\mathfrak{L}_{n} I_{x, n}+\mathfrak{F}_{n} I_{z, n}\right) \mathfrak{I}_{k_{y} k_{y}^{\prime}}\right],
\end{aligned}
$$

where

$$
\begin{aligned}
& \mathfrak{L}_{n} \equiv-\frac{\sqrt{3} A \sqrt{B^{2}-D^{2}}}{|A| B} 2 \operatorname{Im}\left(A_{P, j_{n}}^{E H}\right), \\
& \mathfrak{F}_{n} \equiv \frac{1}{B}\left[(B-D) A_{P, j_{n}}^{E E}+3(B+D) A_{P, j_{n}}^{H H}\right] .
\end{aligned}
$$

In both HF interactions, the edge state spin-flipping terms $I_{ \pm, n} \mathfrak{s}_{k_{y} k_{v}^{\prime}, \mp}$ appear. Moreover, in the HF interaction for $P$-like states, the unusual coupling $I_{x, n} \mathfrak{s}_{k_{y}} k_{y}^{\prime}, z$ is found as discussed in the main text. Note that the terms including the operator $\mathfrak{I}_{k_{y} k_{y}^{\prime}}$ vanish to second order in $k_{y}$ and $k_{y}^{\prime}$ in the position averaging and also in the particle-hole symmetric limit, see Sec. VID.
${ }^{1}$ X.-L. Qi and S.-C. Zhang, Rev. Mod. Phys. 83, 1057 (2011).

${ }^{2}$ C. L. Kane and E. J. Mele, Phys. Rev. Lett. 95, 226801 (2005).

${ }^{3}$ C. L. Kane and E. J. Mele, Phys. Rev. Lett. 95, 146802 (2005).

${ }^{4}$ M. Z. Hasan and C. L. Kane, Rev. Mod. Phys. 82, 3045 (2010).
${ }^{5}$ C. Xu and J. E. Moore, Phys. Rev. B 73, 045322 (2006).

${ }^{6}$ M. König, S. Wiedmann, C. Brüne, A. Roth, H. Buhmann, L. W. Molenkamp, X.-L. Qi, and S.-C. Zhang, Science 318, 766 (2007). 
${ }^{7}$ A. Roth, C. Brüne, H. Buhmann, L. W. Molenkamp, J. Maciejko, X.-L. Qi, and S.-C. Zhang, Science 325, 294 (2009).

${ }^{8}$ M. König, H. Buhmann, L. W. Molenkamp, T. L. Hughes, C.-X. Liu, X. L. Qi, and S. C. Zhang, J. Phys. Soc. Jpn. 77, 031007 (2008).

${ }^{9}$ H. Buhmann, J. Appl. Phys. 109, 102409 (2011).

${ }^{10}$ C. Brüne, A. Roth, H. Buhmann, E. M. Hankiewicz, L. W. Molenkamp, J. Maciejko, X.-L. Qi, and S.-C. Zhang, Nat. Phys. 8, 485 (2012).

${ }^{11}$ M. König, M. Baenninger, A. G. F. Garcia, N. Harjee, B. L. Pruitt, C. Ames, P. Leubner, C. Brüne, H. Buhmann, L. W. Molenkamp, and D. Goldhaber-Gordon, Phys. Rev. X 3, 021003 (2013).

${ }^{12}$ G. M. Gusev, Z. D. Kvon, O. A. Shegai, N. N. Mikhailov, S. A. Dvoretsky, and J. C. Portal, Phys. Rev. B 84, 121302 (2011).

${ }^{13}$ T. L. Schmidt, S. Rachel, F. von Oppen, and L. I. Glazman, Phys. Rev. Lett. 108, 156402 (2012).

${ }^{14}$ J. C. Budich, F. Dolcini, P. Recher, and B. Trauzettel, Phys. Rev. Lett. 108, 086602 (2012).

${ }^{15}$ N. Lezmy, Y. Oreg, and M. Berkooz, Phys. Rev. B 85, 235304 (2012).

${ }^{16}$ F. Crépin, J. C. Budich, F. Dolcini, P. Recher, and B. Trauzettel, Phys. Rev. B 86, 121106 (2012).

${ }^{17}$ J. Maciejko, C. Liu, Y. Oreg, X.-L. Qi, C. Wu, and S.-C. Zhang, Phys. Rev. Lett. 102, 256803 (2009).

${ }^{18}$ A. Ström, H. Johannesson, and G. I. Japaridze, Phys. Rev. Lett. 104, 256804 (2010).

${ }^{19}$ G. Tkachov and E. M. Hankiewicz, Phys. Rev. Lett. 104, 166803 (2010).

${ }^{20}$ J. Maciejko, X.-L. Qi, and S.-C. Zhang, Phys. Rev. B 82, 155310 (2010).

${ }^{21}$ B. Scharf, A. Matos-Abiague, and J. Fabian, Phys. Rev. B 86, 075418 (2012)

${ }^{22}$ P. Delplace, J. Li, and M. Büttiker, Phys. Rev. Lett. 109, 246803 (2012).

${ }^{23}$ M. Kharitonov, Phys. Rev. B 86, 165121 (2012).

${ }^{24}$ G. M. Gusev, A. D. Levin, Z. D. Kvon, N. N. Mikhailov, and S. A. Dvoretsky, Phys. Rev. Lett. 110, 076805 (2013).

${ }^{25}$ B. A. Bernevig, T. L. Hughes, and S.-C. Zhang, Science 314, 1757 (2006).

${ }^{26}$ I. Knez, R.-R. Du, and G. Sullivan, Phys. Rev. Lett. 107, 136603 (2011).

${ }^{27}$ K. Suzuki, Y. Harada, K. Onomitsu, and K. Muraki, Phys. Rev. B 87, 235311 (2013).

${ }^{28}$ L. Du, I. Knez, G. Sullivan, and R.-R. Du, arXiv:1306.1925 (2013).

${ }^{29}$ C. Liu, T. L. Hughes, X.-L. Qi, K. Wang, and S.-C. Zhang, Phys. Rev. Lett. 100, 236601 (2008).

${ }^{30}$ W. A. Coish and J. Baugh, Phys. Status Solidi B 246, 2203 (2009), an excellent review on hyperfine interaction.

${ }^{31}$ J. Schliemann, A. Khaetskii, and D. Loss, J. Phys.: Condens. Matter 15, R1809 (2003).

${ }^{32}$ C. P. Slichter, Principles of Magnetic Resonance (Springer, Berlin, 1996).

${ }^{33}$ A. M. Stoneham, Theory of Defects in Solids: Electronic Structure of Defects in Insulators and Semiconductors (Oxford University Press, Oxford, 1975), Chap. 13.

${ }^{34}$ A. V. Khaetskii, D. Loss, and L. Glazman, Phys. Rev. Lett. 88, 186802 (2002).

${ }^{35}$ F. H. L. Koppens, K. C. Nowack, and L. M. K. Vandersypen, Phys. Rev. Lett. 100, 236802 (2008).
${ }^{36}$ J. R. Petta, A. C. Johnson, J. M. Taylor, E. A. Laird, A. Yacoby, M. D. Lukin, C. M. Marcus, M. P. Hanson, and A. C. Gossard, Science 309, 2180 (2005).

${ }^{37}$ L. Cywiński, Acta Phys. Pol. A 119, 576 (2011).

${ }^{38}$ K. Ono and S. Tarucha, Phys. Rev. Lett. 92, 256803 (2004).

${ }^{39}$ A. Pfund, I. Shorubalko, K. Ensslin, and R. Leturcq, Phys. Rev. Lett. 99, 036801 (2007).

${ }^{40}$ M. S. Rudner and L. S. Levitov, Phys. Rev. Lett. 99, 036602 (2007).

${ }^{41}$ A. M. Lunde, C. López-Monís, I. A. Vasiliadou, L. L. Bonilla, and G. Platero, Phys. Rev. B 88, 035317 (2013).

${ }^{42}$ B. Braunecker, P. Simon, and D. Loss, Phys. Rev. Lett. 102, 116403 (2009).

${ }^{43}$ B. Braunecker, P. Simon, and D. Loss, Phys. Rev. B 80, 165119 (2009).

${ }^{44}$ C. P. Scheller, T.-M. Liu, G. Barak, A. Yacoby, L. N. Pfeiffer, K. W. West, and D. M. Zumbühl, arXiv:1306.1940.

${ }^{45}$ P. Simon and D. Loss, Phys. Rev. Lett. 98, 156401 (2007).

${ }^{46}$ P. Simon, B. Braunecker, and D. Loss, Phys. Rev. B 77, 045108 (2008).

${ }^{47}$ J. Fischer, W. A. Coish, D. V. Bulaev, and D. Loss, Phys. Rev. B 78, 155329 (2008).

${ }^{48}$ J. Fischer and D. Loss, Phys. Rev. Lett. 105, 266603 (2010).

${ }^{49}$ J. Fischer, B. Trauzettel, and D. Loss, Phys. Rev. B 80, 155401 (2009).

${ }^{50}$ J. Fischer, M. Trif, W. Coish, and D. Loss, Solid State Commun. 149, 1443 (2009).

${ }^{51}$ C. Testelin, F. Bernardot, B. Eble, and M. Chamarro, Phys. Rev. B 79, 195440 (2009).

${ }^{52}$ E. A. Chekhovich, M. M. Glazov, A. B. Krysa, M. Hopkinson, P. Senellart, A. Lemaître, M. S. Skolnick, and A. I. Tartakovskii, Nat. Phys. 9, 74 (2013).

${ }^{53}$ M. Dobers, K. v. Klitzing, J. Schneider, G. Weimann, and K. Ploog, Phys. Rev. Lett. 61, 1650 (1988).

${ }^{54}$ K. R. Wald, L. P. Kouwenhoven, P. L. McEuen, N. C. van der Vaart, and C. T. Foxon, Phys. Rev. Lett. 73, 1011 (1994).

${ }^{55}$ J. H. Kim, I. D. Vagner, and L. Xing, Phys. Rev. B 49, 16777 (1994).

${ }^{56}$ D. C. Dixon, K. R. Wald, P. L. McEuen, and M. R. Melloch, Phys. Rev. B 56, 4743 (1997).

${ }^{57}$ E. V. Deviatov, A. Würtz, A. Lorke, M. Yu. Melnikov, V. T. Dolgopolov, D. Reuter, and A. D. Wieck, Phys. Rev. B 69, 115330 (2004).

${ }^{58}$ A. Würtz, T. Müller, A. Lorke, D. Reuter, and A. D. Wieck, Phys. Rev. Lett. 95, 056802 (2005).

${ }^{59}$ T. Nakajima, Y. Kobayashi, and S. Komiyama, Phys. Rev. B 82, 201302 (2010).

${ }^{60}$ T. Nakajima and S. Komiyama, Phys. Rev. B 85, 115310 (2012).

${ }^{61}$ A. M. Lunde and G. Platero, Phys. Rev. B 86, 035112 (2012).

${ }^{62}$ Y. Tanaka, A. Furusaki, and K. A. Matveev, Phys. Rev. Lett. 106, 236402 (2011).

${ }^{63}$ E. Eriksson, A. Ström, G. Sharma, and H. Johannesson, Phys. Rev. B 86, 161103 (2012).

${ }^{64}$ A. Del Maestro, T. Hyart, and B. Rosenow, Phys. Rev. B 87, 165440 (2013).

${ }^{65}$ E. Eriksson, Phys. Rev. B 87, 235414 (2013).

${ }^{66}$ K. Chang and W.-K. Lou, Phys. Rev. Lett. 106, 206802 (2011).

${ }^{67}$ P. Michetti and P. Recher, Phys. Rev. B 83, 125420 (2011). 
${ }^{68}$ L. B. Zhang, F. Cheng, F. Zhai, and K. Chang, Phys. Rev. B 83, 081402 (2011).

${ }^{69}$ W.-Y. Shan, J. Lu, H.-Z. Lu, and S.-Q. Shen, Phys. Rev. B 84, 035307 (2011).

${ }^{70}$ E. Fermi, Zeitschrift für Physik 60, 320 (1930).

${ }^{71}$ Here, $\hbar$ is included explicitly in the gyromagnetic ratios in contrast to, e.g., Fischer et al., ${ }^{47-50}$ but the same sign conventions for the interactions are used.

${ }^{72}$ Many authors simply neglect $r_{c}$ in $h_{i}^{n}(i=2,3)$, since the only role it plays, is to see explicitly that the dipole-dipole-like interaction $h_{2}^{n}$ is zero for an $S$-like state. If $r_{c}$ is absent, then the matrix element of the dipole-dipole-like interaction is not well-defined, see, e.g,. p. 455 in Ref. 33. However, since $r_{c}$ is so small, no nonzero matrix element depends on its magnitude.

${ }^{73} \mathrm{R}$. Winkler, Spin-orbit Coupling Effects in Two-Dimensional Electron and Hole Systems, Springer Tracts in Modern Physics (Springer, Berlin, 2003).

${ }^{74} \mathrm{G}$. Bastard, Wave Mechanics Applied to Semiconductor Heterostructures (EDP Sciences, Paris, 1992).

${ }^{75}$ J. Fabian, A. Matos-Abiague, C. Ertler, P. Stano, and I. Zutic, Acta Physica Slovaca 57, 565 (2007).

${ }^{76}$ D. G. Rothe, R. W. Reinthaler, C.-X. Liu, L. W. Molenkamp, S.-C. Zhang, and E. M. Hankiewicz, New J. Phys. 12, 065012 (2010).

${ }^{77}$ We have chosen the zero of energy such that $\varepsilon_{k}$ is zero at $\mathbf{k}=0$.

${ }^{78}$ B. Zhou, H.-Z. Lu, R.-L. Chu, S.-Q. Shen, and Q. Niu, Phys. Rev. Lett. 101, 246807 (2008).

${ }^{79}$ M. Wada, S. Murakami, F. Freimuth, and G. Bihlmayer, Phys. Rev. B 83, 121310 (2011).

${ }^{80}$ The lattice periodic functions ${ }^{116}\left\langle\mathbf{r} \mid \Gamma_{i}, m_{j}, \mathbf{k}\right\rangle \equiv u_{\mathbf{k}, \Gamma_{i}, m_{j}}(\mathbf{r})$ enter the Bloch states as $\psi_{\mathbf{k}, \Gamma_{i}, m_{j}}(\mathbf{r})=e^{i \mathbf{k} \cdot \mathbf{r}} u_{\mathbf{k}, \Gamma_{i}, m_{j}}(\mathbf{r})$ and have the periodicity of the lattice, i.e., $u_{\mathbf{k}, \Gamma_{i}, m_{j}}\left(\mathbf{r}+\mathcal{R}_{n}\right)=u_{\mathbf{k}, \Gamma_{i}, m_{j}}(\mathbf{r})$ for all Bravais lattice vectors $\boldsymbol{\mathcal { R }}_{n}$.

${ }^{81}$ In the case of a zinc-blende crystal, like HgTe or GaAs, the atomic volume is half of the two-atomic primitive unit cell volume in real space.

${ }^{82}$ Note that there is a slight difference between $\mathbf{R}_{n}$ and $\mathcal{R}_{n}$. The vector $\boldsymbol{\mathcal { R }}_{n}$ is a Bravais lattice vector, ${ }^{116}$ i.e., it points to a specific unit cell, which can consist of several atoms. On the other hand, the vector $\mathbf{R}_{n}$ points to a specific atom. With a suitable choice of Bravais lattice vectors for a two atomic unit cell, the two atoms are at positions: $\mathbf{R}_{n_{1}}=\mathcal{R}_{n^{\prime}}-\mathbf{d} / 2$ and $\mathbf{R}_{n_{2}}=\mathcal{R}_{n^{\prime}}+\mathbf{d} / 2$, where the two atoms are connected by $\mathbf{d}$.

${ }^{83}$ This is in fact an excellent approximation. Fischer et al. ${ }^{47}$ estimated that the contribution to the unit cell integral from nuclear spins not contained in that unit cell to be about two to three orders of magnitude smaller than if the spin is inside the unit cell, see Appendix C in Ref. 47.

${ }^{84}$ M. Gueron, Phys. Rev. 135, A200 (1964).

${ }^{85}$ The relative signs between the atomic wave functions stem from the bonding and antibonding nature of the bands, i.e., the $S$-like band is antibonding and the $P$-like bands are bonding. ${ }^{75}$ However, these signs do not play a role here, since they do not enter in the matrix elements of the HF interactions on the present level of approximation.

${ }^{86}$ Note that we keep the factor $e^{i\left(\mathbf{k}^{\prime}-\mathbf{k}\right) \cdot \mathcal{R}_{n \perp}} / L_{x} L_{y}$ outside the definition of the position dependent HF coupling in order to have $A_{j_{n}}^{S}\left(\mathcal{Z}_{n}\right)$ independent of $\mathbf{k}$. However, this choice means that the unit of $A_{j_{n}}^{S}\left(\mathcal{Z}_{n}\right)$ is not energy but energy times length squared.

${ }^{87}$ The prefactor of $1 / 2$ is the spin projection of the electron on the $z$ axis and therefore not included in $A_{j_{n}}^{S}\left(\mathcal{Z}_{n}\right)$ in order to have the
Hamiltonian in a form analog to the quantum dot case, where $H=\sum_{n} A_{n} \mathbf{S} \cdot \mathbf{I}_{n}$.

${ }^{88}$ The parity $\mathcal{P}$ around the atomic core for the atomic-like wave functions $\Psi_{\Gamma_{6}, m_{j}}^{\mathrm{Hg} / \mathrm{Te}}$ is even ( $S$-like), whereas $\Psi_{\Gamma_{8}, m_{j}}^{\mathrm{Hg} / \mathrm{Te}}$ has odd parity ( $P$-like). This follows, e.g., from the parity of the spherical harmonics. Since the dipole-dipole interaction is invariant under atomic parity, $\mathcal{P} h_{2}^{n} \mathcal{P}^{-1}=+h_{2}^{n}$, the matrix elements between $S$ and $P$-like states vanish.

${ }^{89}$ For instance, we could choose $r_{\max }$ such that half of the unit cell volume is in a sphere around the $\mathrm{Hg}$ atom, i.e., $v_{u c} / 2=4 \pi r_{\max }^{3} / 3$, or $r_{\max }$ could be chosen to be equal to half the distance between the atoms $|\mathbf{d}| / 2$. However, in either case, $r_{\max }$ is larger then $a_{0}$ such that the numerical value of the integral do not change-even if $r_{\max }$ is set to infinity.

${ }^{90}$ In principle, an atomic HF coupling could be introduced for $h_{2}^{n}$ and $h_{3}^{n}$ separately. The only difference between the two would be their normalization constants $N_{\Gamma_{8}, m_{j}}$ with $m_{j}=\frac{3}{2}, \frac{1}{2}$, respectively. However, due to their numerical similarity, $N_{\Gamma_{8}, 3 / 2} \simeq N_{\Gamma_{8}, 1 / 2}$, we consider this to be an unnecessary complication in the case of HgTe.

${ }^{91}$ D. G. Rothe, E. M. Hankiewicz, B. Trauzettel, and M. Guigou, Phys. Rev. B 86, 165434 (2012).

${ }^{92}$ In practices, the envelope functions can often be found by requiring them to vanish at the boundary of a certain region. ${ }^{66,67,69,78}$ Alternatively, one can model a boundary with a sign change in the Dirac mass $M_{0}$ on either side of a boundary. ${ }^{117,118}$ Other models with only linear derivatives can require other methods of confinement. ${ }^{19,119,120}$

${ }^{93}$ The normalization of the envelope functions is $\int d \mathbf{r}_{\perp} \sum_{\zeta=E \pm, H \pm}\left|\phi_{\eta, \zeta}\left(\mathbf{r}_{\perp}\right)\right|^{2}=1$, which can be showed along the lines of Appendix B.

${ }^{94} \mathrm{~A} 70$ - $\AA$-thick HgTe quantum well is a $2 \mathrm{D}$ TI with the $\mathrm{BHZ}$ model parameters: ${ }^{1} A=3.65 \mathrm{eV} \AA, B=-68.6 \mathrm{eV} \AA^{2}, D=$ $-51.2 \mathrm{eV} \AA^{2}$, and $M_{0}=-0.010 \mathrm{eV}$.

${ }^{95}$ For $M_{0} / B$ larger than $A^{2} /\left(4 B^{2}\right)$ the parameters $\lambda_{1,2}$ can get an imaginary part, which leads to an oscillatory decay of the transverse edge states into the bulk part of the 2D TI. See, e.g., Ref. 121 or the Appendix in Ref. 122 for a discussion in the particle-hole symmetric case $(D=0)$.

${ }^{96}$ J. J. Sakurai, Modern Quantum Mechanics (Addison Wesley, Reading, MA, 1993).

${ }^{97} \mathrm{H}$. Bruus and K. Flensberg, Many-body Quantum Theory in Condensed Matter Physics, 1st ed. (Oxford University Press, Denmark, 2004).

${ }^{98}$ A. Khaetskii, D. Loss, and L. Glazman, Phys. Rev. B 67, 195329 (2003).

${ }^{99}$ This estimate is based on a 7-nm-thick quantum well with a HES width of about $W_{x} \sim \lambda_{2}^{-1} \simeq 50 \mathrm{~nm}$ for $k=0$ and using that the atomic volume $v_{a}$ is half of the Wigner-Seitz unit cell volume, i.e., $v_{a}=8|\mathbf{d}|^{3} /(3 \sqrt{3}) \simeq 3.3 \times 10^{-2} \mathrm{~nm}^{3}$ for the zinc-blende crystal, where $|\mathbf{d}|=0.279 \mathrm{~nm}$ is the bond length between the $\mathrm{Hg}$ and $\mathrm{Te}$ atoms. ${ }^{33}$

${ }^{100}$ The definition (26) of the atomic $P$-like HF couplings $A_{P, j_{n}}^{\text {Atomic }}$ includes the factor $\left(N_{\Gamma_{8}}\right)^{2}\left|\alpha_{j_{n}}\right|^{2}$ in contrast to the definition in Eq. (7) of Ref. 47. However, the definition (18) of the atomic contact HF coupling is identical to the one in Ref. 47, since they both include the factor $\left(N_{\Gamma_{6}}\right)^{2}\left|\alpha_{j_{n}}\right|^{2}$. Therefore some care has to be taken in the comparison of our results to those in Ref. 47.

${ }^{101}$ A. W. Overhauser, Phys. Rev. 92, 411 (1953). 
${ }^{102}$ I. A. Merkulov, A. L. Efros, and M. Rosen, Phys. Rev. B 65, 205309 (2002)

${ }^{103}$ S. I. Erlingsson and Y. V. Nazarov, Phys. Rev. B 66, 155327 (2002).

${ }^{104}$ Using a Gaussian distributed semiclassical field from the nuclear spins, ${ }^{102,103}$ we can derive the ensemble averaged energy gap to be approximately $E_{g} \simeq \sqrt{\frac{\pi}{6}} \frac{1}{\sqrt{N}} A_{\text {eff }}^{\perp}$. Here, the effective in-plane HF coupling constant is given by $A_{\text {eff }}^{\perp} \equiv \sqrt{\sum_{j} \frac{N_{j}}{N}\left(A_{j}^{\perp}\right)^{2} \mathcal{I}_{j}\left(\mathcal{I}_{j}+1\right)}$, where the sum is over the nuclear isotope types $j={ }^{199} \mathrm{Hg},{ }^{201} \mathrm{Hg}$, ${ }^{123} \mathrm{Te},{ }^{125} \mathrm{Te}, \mathcal{I}_{j}$ is the nuclear spin quantum number [see Eq. (3)] and $N_{j}$ is the number of nuclear spins of isotope $j$ covered by the HESs.

${ }^{105}$ P. Y. Yu and M. Cardona, Fundamentals of Semiconductors, 3rd ed. (Springer, Berlin, 2001).

${ }^{106}$ Even though the spherical harmonic ${ }^{96} Y_{m=0}^{l=0}=1 / \sqrt{4 \pi}$ corresponding to the $|S\rangle$ state is real, the state $|S\rangle$ is often conventionally chosen to be purely imaginary, see, e.g., Table $\mathrm{C} 1$ in Appendix $\mathrm{C}$ of Ref. 73. Sometimes an imaginary unit $i$ is actually included explicitly and not absorbed into the notation of $|S\rangle$ as it is done here, see, e.g., Chuang ${ }^{123}$ pp. 131, 634, and 635 [see Eq. (A32)] or Ref. 74. Generally, it is convenient to have $|S\rangle$ purely imaginary such that the parameter $P_{0}=\left(\hbar / m_{0}\right)\left\langle S\left|\left(-i \hbar \partial_{x}\right)\right| P_{x}\right\rangle$ connecting $S$ - and $P$-like bands in $\mathbf{k} \cdot \mathbf{p}$ theory becomes real, since the $P$-like states are real. However, the choice of having $|S\rangle$ purely imaginary affects the time-reversal properties of the $|E \pm\rangle$ states.

${ }^{107}$ E. G. Novik, A. Pfeuffer-Jeschke, T. Jungwirth, V. Latussek, C. R. Becker, G. Landwehr, H. Buhmann, and L. W. Molenkamp, Phys. Rev. B 72, 035321 (2005).

${ }^{108}$ This overall sign-change compared to the conventional one ${ }^{73}$ does not come from the spherical harmonics as given by Sakurai, ${ }^{96}$ see, e.g., Winkler. ${ }^{73}$ For many purposes, this sign does not play a role, since it is simply an overall phase factor. However, the matrix elements between $\Gamma_{6}$ and $\Gamma_{8}$ states change sign. This is taken care of in Refs. 25 and 107 by changing the sign of the definition of the $P_{0}$ parameter in the $\mathbf{k} \cdot \mathbf{p}$ theory, so the $\mathbf{k} \cdot \mathbf{p}$
Hamiltonian looks formally the same. Therefore Refs. 25 and 76 use $P_{0}=-\frac{\hbar}{m_{0}}\left\langle S\left|p_{x}\right| P_{x}\right\rangle$ with the opposite sign compared to the convention in Winkler, ${ }^{73}$ where $P_{0}=+\frac{\hbar}{m_{0}}\left\langle S\left|p_{x}\right| P_{x}\right\rangle$. Note that this sign has no effect on the time-reversal properties of the states.

${ }^{109}$ G. Katsaros, V. N. Golovach, P. Spathis, N. Ares, M. Stoffel, F. Fournel, O. G. Schmidt, L. I. Glazman, and S. De Franceschi, Phys. Rev. Lett. 107, 246601 (2011).

${ }^{110}$ J. M. Luttinger, Phys. Rev. 102, 1030 (1956).

${ }^{111}$ Dietrich G. Rothe (private communication).

${ }^{112}$ A. Willig and B. Sapoval, J. Phys. Lett. 38, 57 (1977).

${ }^{113} \mathrm{We}$ remark that this is a number with some uncertainty in the literature. For instance, Harrison ${ }^{124}$ gives $\alpha_{\mathrm{Te}} \simeq \sqrt{0.9}$ (and $\alpha_{\mathrm{Hg}} \simeq$ $\sqrt{0.1}$ ). However, for the present estimate, we use the value by Willig and Sapoval in Ref. 112.

${ }^{114}$ E. Clementi and D. L. Raimondi, J. Chem. Phys. 38, 2686 (1963).

${ }^{115}$ E. Clementi, D. L. Raimondi, and W. P. Reinhardt, J. Chem. Phys. 47, 1300 (1967)

${ }^{116}$ N. W. Ashcroft and N. D. Mermin, Solid State Physics (Thomson Learning, New York, 1976).

${ }^{117}$ S. Murakami, S. Iso, Y. Avishai, M. Onoda, and N. Nagaosa, Phys. Rev. B 76, 205304 (2007).

${ }^{118}$ P. Michetti, P. H. Penteado, J. C. Egues, and P. Recher, Semicond. Sci. Technol. 27, 124007 (2012).

${ }^{119}$ M. Berry and R. J. Mondragon, Proc. R. Soc. London A 412, 53 (1987).

${ }^{120}$ J. A. Fürst, J. G. Pedersen, C. Flindt, N. A. Mortensen, M. Brandbyge, T. G. Pedersen, and A.-P. Jauho, New J. Phys. 11, 095020 (2009).

${ }^{121}$ E. B. Sonin, Phys. Rev. B 82, 113307 (2010).

${ }^{122}$ K.-I. Imura, A. Yamakage, S. Mao, A. Hotta, and Y. Kuramoto, Phys. Rev. B 82, 085118 (2010).

${ }^{123}$ S. L. Chuang, Physics of Optoelectronic Devices, 1st ed. (WileyInterscience, New York, 1999).

${ }^{124}$ W. A. Harrison, Phys. Rev. B 8, 4487 (1973). 